\title{
ROBUST EQUILIBRIUM INVESTMENT AND REINSURANCE STRATEGY WITH BOUNDED MEMORY AND COMMON SHOCK DEPENDENCE
}

\author{
SHENG Li*(1)
}

\begin{abstract}
In this paper, we consider the robust investment and reinsurance problem with bounded memory and risk co-shocks under a jump-diffusion risk model. The insurer is assumed to be ambiguityaverse and make the optimal decision under the mean-variance criterion. The insurance market is described by two-dimensional dependent claims while the risky asset is depicted by the jump-diffusion model. By introducing the performance in the past, we derive the wealth process depicted by a stochastic delay differential equation (SDDE). Applying the stochastic control theory under the game-theoretic framework, together with stochastic control theory with delay, the robust equilibrium investmentreinsurance strategy and the corresponding robust equilibrium value function are derived. Furthermore, some numerical examples are provided to illustrate the effect of market parameters on the optimal investment and reinsurance strategy.
\end{abstract}

Mathematics Subject Classification. 62P05, 91B30, 93E20.

Received April 22, 2021. Accepted December 14, 2021.

\section{INTRODUCTION}

In the insurance market, insurers improve the economic efficiency of the system by spreading individual risks. The policyholder transfers the risk to insurers by paying premiums and insurers are free to use the premiums for investment. It is necessary to enter the reinsurance market when the risk it insures exceeds its limits. In the reinsurance market, by paying reinsurance fees, the insured business is partially transferred to other insurers, to achieve the purpose of risk diversification, loss control, and stable operation. As insurance for insurance, reinsurance is a very important part of the overall insurance system. The core issue of insurers is risk management and control, that is, the management and control of the surplus process of insurers, combining the actuarial problems of insurance and investment portfolio problem, not only to focus on the risks and returns of investment, but also to focus on the company's underwriting risks, to ensure that it can pay the claims of policyholders in a timely manner. As a result, the issue of investment and reinsurance by insurance companies has attracted a great deal of attention in recent years. Browne [9] investigates firstly the optimal investment strategy for an insurer under maximizing exponential utility function and minimizing the ruin probability. Højgaard and Taksar [15] study the proportional reinsurance problem with the maximum return function under the diffusion approximation (DA) model and obtain an approximate expression for the optimal strategy and the

Keywords. Robust investment and reinsurance, mean-variance, jump-diffusion, two-dimensional claim, game theory.

School of Statistics, Chengdu University of Information Technology, Chengdu 610103, P.R. China.

*Corresponding author: lisheng@cuit.edu.cn 
value function. Hipp and Plum [14] study the optimal investment strategy that minimizes the ruin probability of insurer under the classical Cramer-Lundberg (C-L) model. Yang and Zhang [24] consider the optimal investment problem under the jump-diffusion model under maximizing expected utility. Bai and Guo [2] study the optimal investment strategy of an insurer investing in multiple risk assets under the DA model. Cao and Wan [10] obtain optimal investment and reinsurance strategy under maximizing the expected utility of terminal wealth.

In addition, the mean-variance criterion is another goal of great interest for insurers since it takes into account not only the risk but also the returns. Due to its rationality and practicality, the mean-variance has become a popular decision criterion in financial theory. The study of the mean-variance can be traced back to Markowitz [19]. Since then, mean-variance has been extensively studied. Li and $\mathrm{Ng}$ [16] introduce an embedding technique to transform the mean-variance problem into a stochastic linear quadratic control problem in a discrete-time model, which was extended to the corresponding continuous-time model by Zhou [29]. See also Bäuerle [4], Bai and Zhang [3], Bi and Guo [5] for the application of the mean-variance criterion to investment and reinsurance problem. However, it is worth noting that the dynamic mean-variance criterion lacks iteratedexpectation property, which leads to time-inconsistent in the sense that the Bellman optimality principle does not hold anymore. In fact, time-consistency of the optimal strategy is a fundamental requirement for rational decision making in many situations. Accordingly, Björk and Murgoci [6] and Björk et al. [7] develop a general theory for Markovian time-inconsistent stochastic control problems. They obtain a time-consistent equilibrium strategy that is not only optimal at the current time but also optimal in the future. In recent years, there has been an increased interest in finding a time-consistent equilibrium strategy for the mean-variance investment and reinsurance problem. Applying this theory, Zeng and Li [26], Zeng et al. [27], Lin and Qian [17] derive the optimal time-consistent investment and reinsurance strategy for the mean-variance insurer.

However, there are three aspects of the literature mentioned above that deserve further exploration. Firstly, they are the lack of consideration of model uncertainty. Maenhout [18] investigates the effect of ambiguity on the intertemporal portfolio choice in a setting with constant investment opportunities and in a setting with a mean-reverting equity risk premium, respectively. Secondly, the correlation between insurance operations is not considered. In the real market, different insurance businesses often have some common shocks. For instance, the outbreak of the 2019-nCoV may lead to the simultaneous occurrence of medical claims and death claims. Besides, auto insurance/third party insurance, casualty insurance/health insurance, life insurance/endowment insurance often have also interdependent risk shocks. To demonstrate the interdependence between different insurance businesses, a risk dependence model is proposed. Third, the influence of past information on insurers' decisions is not incorporated into the model. Actually, insurers pay attention not only to the current stock price but also to the stock price trend in the past period when investing in stocks. Also, the past information always impacts the decision-making of insurers, who tend to examine performance trends over time, not just current financial information, when deciding how much reinsurance to purchase. Considering the past information in the model helps us make more rational decisions. In this paper, we formulate a robust optimization problem with alternative models and establish the corresponding extended Hamilton-Jacobi-Bellman (HJB) system of equations. Furthermore, we derive both the robust equilibrium reinsurance-investment strategy and the corresponding equilibrium value function. Some special cases of our model are also provided, which show that our model and results extend some ones in the existing literature. Finally, the economic implications of our findings are illustrated, and utility losses from ignoring model uncertainty, jump risks and prohibiting reinsurance are analyzed using numerical examples. The main contributions of this paper are as follows: (1) We incorporate past performance into the robust investment-reinsurance problem where the insurer's optimal decision in the worstcase model is based on a weighting of past and present information. (2) Two insurance businesses with common shocks are studied and two robust reinsurance strategies which are influenced by some common factor are analyzed. (3) The analytical solution is obtained for the robust equilibrium investment and reinsurance strategy when there is a jump process in the price of risky assets. (4) Utility losses from ignoring model uncertainty, jump risks and prohibiting reinsurance for the AAI are analyzed, and some new findings are provided.

The remainder of this paper is organized as follows. Section 2 formulates the mean-variance investment and reinsurance problem with bounded memory and common shock dependence under model uncertainty. In 
Section 3, by solving the extended HJB equation, we derive the robust equilibrium investment and reinsurance strategy and the corresponding equilibrium value functions. In Section 4, we present a numerical example for analyzing the impact of changes in the model parameter on a robust equilibrium strategy. Section 5 concludes this paper.

\section{General Formulation}

In this paper, we consider a filtered complete probability space $\left(\Omega, \mathcal{F},\left\{\mathcal{F}_{t}\right\}_{t \in[0, T]}, \mathbb{P}\right)$ satisfying the usual conditions, i.e., $\left\{\mathcal{F}_{t}\right\}_{t \in[0, T]}$ is right continuous and $\mathbb{P}$-complete, where $\left\{\mathcal{F}_{t}\right\}_{t \in[0, T]}$ is the information of the market available up to time $t,[0, T]$ is a fixed and finite time horizon. All stochastic processes introduced below are assumed to be well-defined and adapted processes in this probability space, and we assume that all stochastic processes are separable. In addition, we suppose that there are no transaction costs or taxes in the financial or the insurance market, and trading can be continuously done.

Suppose an insurer has an insurance portfolio business, which is composed of two different insurance businesses, such as medical insurance and death insurance. Suppose that the random variables $\left\{Y_{1 i}, i \geq 1\right\}$ represent the claim amount of the first type of insurance business, they are independent of each other and have the same distribution function $F_{1}\left(y_{1}\right) .\left\{Y_{2 i}, i \geq 1\right\}$ represent the claim amount of the second type of insurance business, they are mutually independent and have the common distribution function $F_{2}\left(y_{2}\right)$. We assume that if $y_{1} \leq 0$, then $F_{1}\left(y_{1}\right)=0$; otherwise $0<F_{1}\left(y_{1}\right) \leq 1$. And we also assume that if $y_{2} \leq 0$, then $F_{2}\left(y_{2}\right)=0$; otherwise $0<F_{2}\left(y_{2}\right) \leq 1$. In addition, their moment generating functions $M_{Y_{1}}(\iota)$ and $M_{Y_{2}}(\iota)$ exist. The cumulative claim process of the two insurance businesses are as follows.

$$
C_{1}(t)=\sum_{i=1}^{\widetilde{N}_{1}(t)} Y_{1 i}, \quad C_{2}(t)=\sum_{i=1}^{\tilde{N}_{2}(t)} Y_{2 i}
$$

where $\left\{\tilde{N}_{1}(t)\right\}_{t>0}$ and $\left\{\tilde{N}_{2}(t)\right\}_{t>0}$ represent the number of claims for the first and second categories of insurance business up to time $t$, respectively.

For different insurance businesses, it is assumed that they are interdependent as follows.

$$
\widetilde{N}_{1}(t)=N_{1}(t)+N(t), \quad \widetilde{N}_{2}(t)=N_{2}(t)+N(t),
$$

where $\{N(t)\}_{t>0},\left\{N_{1}(t)\right\}_{t>0}$ and $\left\{N_{2}(t)\right\}_{t>0}$ are three independent Poisson processes, the corresponding intensities are $\lambda, \lambda_{1}$ and $\lambda_{2}$. Therefore, the total claim amount of these two types of the insurance business is

$$
C(t)=C_{1}(t)+C_{2}(t)=\sum_{i=1}^{N_{1}(t)+N(t)} Y_{1 i}+\sum_{i=1}^{N_{2}(t)+N(t)} Y_{2 i}
$$

Suppose for arbitrary $\iota \in(0, \bar{\iota}), E\left[Y_{1 i} e^{\iota Y_{1 i}}\right]$ and $E\left(Y_{2 i} e^{\iota Y_{2 i}}\right)$ exist. And, for some $\bar{\iota} \in(0, \infty]$, there are $\lim _{\iota \rightarrow \bar{\iota}} E\left[Y_{1 i} e^{\iota Y_{1 i}}\right] \rightarrow \infty$ and $\lim _{\iota \rightarrow \bar{\iota}} E\left[Y_{2 i} e^{\iota Y_{2 i}}\right] \rightarrow \infty$.

For simplicity, we define

$$
\begin{array}{ll}
a_{1}:=E\left[C_{1}(t)\right]=\left(\lambda+\lambda_{1}\right) \mu_{11}, & b_{1}^{2}:=\operatorname{Var}\left[C_{1}^{2}(t)\right]=\left(\lambda+\lambda_{1}\right) \mu_{12}, \\
a_{2}:=E\left[C_{2}(t)\right]=\left(\lambda+\lambda_{2}\right) \mu_{21}, & b_{2}^{2}:=\operatorname{Var}\left[C_{2}^{2}(t)\right]=\left(\lambda+\lambda_{2}\right) \mu_{22},
\end{array}
$$

where $\mu_{11}=E\left[Y_{1 i}\right], \mu_{12}=E\left[Y_{1 i}^{2}\right], \mu_{21}=E\left[Y_{2 i}\right]$ and $\mu_{22}=E\left[Y_{2 i}^{2}\right]$. According to Grandell [13] and Schmidli [21], $C_{1}(t)$ and $C_{2}(t)$ can be approximated by the Brownian motion $\widehat{C}_{1}(t)$ and $\widehat{C}_{2}(t)$ with drift, respectively.

$$
\widehat{C}_{1}(t)=a_{1} t-b_{1} W_{1}(t), \quad \widehat{C}_{2}(t)=a_{2} t-b_{2} W_{2}(t),
$$


where $\left\{W_{1}(t)\right\}_{t \in[0, T]}$ and $\left\{W_{2}(t)\right\}_{t \in[0, T]}$ are standard Brownian motion with $E\left(W_{1}(t) W_{2}(t)\right)=\rho t$, where $\rho=$ $\frac{\lambda \mu_{11} \mu_{21}}{b_{1} b_{2}}$. The surplus process of the insurer up to time $t$ is modeled by

$$
R(t)=R_{0}+c_{0} t-\widehat{C}_{1}(t)-\widehat{C}_{2}(t)
$$

where $R_{0}$ is the initial surplus, and $c_{0}$ is the premium rate. We assume that insurance premium rate at time $t$ be calculated by the expected value principle, that is, $c_{0}=\left(1+\theta_{1}\right) a_{1}+\left(1+\theta_{2}\right) a_{2}$, where $\theta_{1}$ and $\theta_{2}$ are the safety loadings of the insurer for the first class claims and second class claims, respectively. In addition, the insurer can continuously purchase proportional reinsurance. And $q_{1}(t)$ and $q_{2}(t)$ represent the insurer's retention level after purchasing reinsurance, respectively. That is to say, the insurer pays $q_{1}(t) Y_{1 i}$ (or $q_{2}(t) Y_{2 i}$ ) of a claim occurring at time $t$ and the reinsurer pays $1-q_{1}(t) Y_{1 i}$ (or $\left.1-q_{2}(t) Y_{2 i}\right)$. Let the reinsurance premium also be calculated by the expected value principle. For the new business, the premium has to be paid at rate $\left(1-q_{1}(t)\right)\left(1+\eta_{1}\right) a_{1}+\left(1 q_{2}(t)\right)\left(1+\eta_{2}\right) a_{2}$, where $\eta_{1}$ and $\eta_{2}$ are the safety loadings of the reinsurer for the first class claims and second class claims, respectively. To exclude the insurer's arbitrage behavior, we assume that $\eta_{1}>\theta_{1}, \eta_{2}>\theta_{2}$. Note that for the insurer, $q_{1}(t) \in[0,1]\left(q_{2}(t) \in[0,1]\right)$ corresponds to a reinsurance cover and $q_{1}(t)>1\left(q_{2}(t)>1\right)$ would mean that the company can take an extra insurance business from other companies (i.e., act as a reinsurer for other cedents). After reinsurance, the premium of the insurer is given by

$$
\begin{aligned}
c & =c_{0}-\left[\left(1-q_{1}(t)\right)\left(1+\eta_{1}\right) a_{1}+\left(1-q_{2}(t)\right)\left(1+\eta_{2}\right) a_{2}\right] \\
& =\left[\left(1+\eta_{1}\right) q_{1}(t)+\theta_{1}-\eta_{1}\right] a_{1}+\left[\left(1+\eta_{2}\right) q_{2}(t)+\theta_{2}-\eta_{2}\right] a_{2} .
\end{aligned}
$$

Then the reserve process $\{R(t)\}_{t \geq 0}$ of the insurer is

$$
\begin{aligned}
\mathrm{d} R(t)= & c d t-q_{1}(t) d \widehat{C}_{1}(t)-q_{2}(t) d \widehat{C}_{2}(t) \\
= & {\left[a_{1} \eta_{1} q_{1}(t)+\left(\theta_{1}-\eta_{1}\right) a_{1}+a_{2} \eta_{2} q_{2}(t)+\left(\theta_{2}-\eta_{2}\right) a_{2}\right] \mathrm{d} t } \\
& +b_{1} q_{1}(t) \mathrm{d} W_{1}(t)+b_{2} q_{2}(t) \mathrm{d} W_{2}(t) \\
= & {\left[a_{1} \eta_{1} q_{1}(t)+\left(\theta_{1}-\eta_{1}\right) a_{1}+a_{2} \eta_{2} q_{2}(t)+\left(\theta_{2}-\eta_{2}\right) a_{2}\right] \mathrm{d} t } \\
& +\sqrt{b_{1}^{2} q_{1}^{2}(t)+b_{2}^{2} q_{2}^{2}(t)+2 q_{1}(t) q_{2}(t) \lambda \mu_{11} \mu_{21}} \mathrm{~d} W_{0}(t),
\end{aligned}
$$

where $W_{0}(t)$ is a standard Brownian motion.

We consider a financial market consisting of a risk-free asset and a risky asset, in which financial assets can be traded continuously. The dynamic evolution of a risk-free asset price is given by

$$
\mathrm{d} S_{0}(t)=r S_{0}(t) \mathrm{d} t, \quad S_{0}(0)=1,
$$

where $r(>0)$ is the interest rate of the risk-free asset. The price dynamics of the risky asset is described by the following process:

$$
\mathrm{d} S(t)=S(t-)\left[\alpha \mathrm{d} t+\sigma \mathrm{d} W(t)+d \sum_{i=1}^{N_{0}(t)} Y_{0 i}\right], \quad S(0)=s_{0},
$$

where $\alpha$ denotes the appreciation rate, $\sigma$ represents the volatility coefficients for Brownian motion. And $\{W(t)\}_{t \in[0, T]}$ is a standard $\{\mathcal{F}\}_{t \geq 0}$-adapted Brownian motion. $\left\{N_{0}(t)\right\}_{t>0}$ is a Poisson process with parameter $\lambda_{0}$ and the jump $\left\{Y_{0 i}, i \geq 1\right\}$ is a set of independent random variables with the same distribution as $F_{0}\left(y_{0}\right)$. We suppose that $0<F_{0}\left(y_{0}\right) \leq 1$ for $y_{0}>-1$; otherwise $F_{0}\left(y_{0}\right)=0$. And let $E\left[Y_{0 i}\right]=\mu_{01}, E\left[Y_{0 i}^{2}\right]=\mu_{02}$. Moreover, we assume that $\{W(t)\}_{t \in[0, T]}$ and $\left\{W_{0}(t)\right\}_{t \in[0, T]}$ are mutually independent. And in order to exclude risk-free arbitrage in financial markets, we assume that $\alpha+\lambda_{0} \mu_{01}>r$. 
Next, we consider a Poisson random measure $N_{0}(\cdot, \cdot)$ on $\Omega \times[0, T] \times(-1, \infty)$ to represent the compound Poisson process $\sum_{i=1}^{N_{0}(t)} Y_{0 i}$ as

$$
\sum_{i=1}^{N_{0}(t)} Y_{0 i}=\int_{0}^{t} \int_{-1}^{\infty} y_{0} N_{0}\left(\mathrm{~d} s, \mathrm{~d} y_{0}\right), \quad t \in[0, T] .
$$

If we denote by $\nu\left(\mathrm{d} t, \mathrm{~d} y_{0}\right)=\lambda_{0} \mathrm{~d} t \mathrm{~d} F_{0}\left(y_{0}\right)$, then

$$
E\left[\sum_{i=1}^{N_{0}(t)} Y_{0 i}\right]=\int_{0}^{t} \int_{-1}^{\infty} y_{0} \nu\left(\mathrm{d} s, \mathrm{~d} y_{0}\right), \quad t \in[0, T]
$$

where $\nu(\cdot, \cdot)$ is the compensators of the random measure. Hence the compensated measure $\widetilde{N}_{0}(\cdot, \cdot)=N_{0}(\cdot, \cdot)-$ $\nu(\cdot, \cdot)$ is related to the compound Poisson process $\sum_{i=1}^{N_{0}(t)} Y_{0 i}$ as follows:

$$
\int_{0}^{t} \int_{-1}^{\infty} y_{0} \widetilde{N}_{0}\left(\mathrm{~d} s, \mathrm{~d} y_{0}\right)=\sum_{i=1}^{N_{0}(t)} Y_{0 i}-E\left[\sum_{i=1}^{N_{0}(t)} Y_{0 i}\right], \quad t \in[0, T] .
$$

Assume that $X(t)$ denotes the wealth of the insurer at time $t \in[0, T], p_{1}(t)$ denotes the amount invested in the risky asset, then $X(t)-p_{1}(t)$ is the investment amount of a risk-free asset. Define $\pi(t)=\left(p_{1}(t), q_{1}(t), q_{2}(t)\right)$ as the investment-reinsurance strategy at time $t$, and consider the impact of the historical performance $f(t, X(t)-$ $\bar{L}(t), X(t)-M(t))$, then we have the following wealth dynamics depicted by a stochastic delay differential equation (SDDE):

$$
\begin{aligned}
\mathrm{d} X^{\pi}(t)= & \left(X^{\pi}(t)-p_{1}(t)\right) \frac{\mathrm{d} S_{0}(t)}{S_{0}(t)}+p_{1}(t) \frac{\mathrm{d} S(t)}{S(t)}+\mathrm{d} R(t)-f(t, X(t)-\bar{L}(t), X(t)-M(t)) \\
= & {\left[\left(r-\gamma_{1}-\gamma_{2}\right) X^{\pi}(t)+\bar{\gamma}_{1} L^{\pi}(t)+\gamma_{2} M^{\pi}(t)+(\alpha-r) p_{1}(t)\right.} \\
& \left.+\left(\theta_{1}-\eta_{1}+q_{1}(t) \eta_{1}\right) a_{1}+\left(\theta_{2}-\eta_{2}+q_{2}(t) \eta_{2}\right) a_{2}\right] \mathrm{d} t \\
& +\sqrt{b_{1}^{2} q_{1}^{2}(t)+b_{2}^{2} q_{2}^{2}(t)+2 q_{1}(t) q_{2}(t) \lambda \mu_{11} \mu_{21}} \mathrm{~d} W_{0}(t)+p_{1}(t) \sigma \mathrm{d} W(t) \\
& +\int_{-1}^{\infty} p_{1}(t) y_{0} N_{0}\left(\mathrm{~d} t, \mathrm{~d} y_{0}\right) .
\end{aligned}
$$

Following Shen and Zeng [22], in equation (2.3) above, we suppose that

$$
\begin{aligned}
f(t, X(t)-\bar{L}(t), X(t)-M(t)) & =\gamma_{1}(X(t)-\bar{L}(t))+\gamma_{2}(X(t)-M(t)) \\
& =\gamma_{1}\left(X(t)-\frac{L(t)}{\int_{-h}^{0} e^{A u} \mathrm{~d} u}\right)+\gamma_{2}(X(t)-M(t)) \\
& =\left(\gamma_{1}+\gamma_{2}\right) X(t)-\bar{\gamma}_{1} L(t)-\gamma_{2} M(t)
\end{aligned}
$$

where $\gamma_{1}(\geq 0)$ and $\gamma_{2}(\geq 0)$ are constants. And obviously, $\bar{\gamma}_{1}=\frac{\gamma_{1}}{\int_{-h}^{0} e^{A u} \mathrm{~d} u} \cdot L(t)=\int_{-h}^{0} e^{A u} X^{\pi}(t+u) \mathrm{d} u, \bar{L}(t)=$ $\frac{L(t)}{\int_{-h}^{0} e^{A u} \mathrm{~d} u}$ and $M(t)=X(t-h)$ represent the integrated, average and point by point delay information during time $[t-h, t]$, respectively. And $A(\geq 0)$ and $h(\geq 0)$ are given average parameter and delay parameter, respectively. Note that $\bar{L}(t)$ is defined as the weighted average value of wealth process $X(\cdot)$ in the time interval $[t-h, t]$, and the 
exponential decay factor $e^{A u}(u \in[-h, 0])$ denotes the weight. Moreover, We assume that $X(t)=X_{0}, \forall t \in[-h, 0]$, which can be interpreted as that the insurer owns wealth $X_{0}$ at time $-h$ and does not carry out any business operation within $[-h, 0]$. The integrated delay wealth initial value can be calculated by $L(0)=\frac{X_{0}}{A}\left(1-e^{-A h}\right)$.

To take historical operating performance into account, the insurer will focus on both terminal wealth $X^{\pi}(T)$ and historical average operating performance $\bar{L}^{\pi}(T)$, hence we formulate the optimal investment and reinsurance problem with delay under the mean-variance criterion as follows:

$$
\sup _{\pi \in \Pi}\left\{E_{t, x, l}\left[X^{\pi}(T)+\bar{\beta} \bar{L}^{\pi}(T)\right]-\frac{\omega}{2} \operatorname{Var}_{t, x, l}\left[X^{\pi}(T)+\bar{\beta} \bar{L}^{\pi}(T)\right]\right\},
$$

where $\omega$ is the risk averse coefficient. The delay parameter $\bar{\beta}(\in[0,1])$ is a constant. $E_{t, x, l}[\cdot]$ and $\operatorname{Var}_{t, x, l}[\cdot]$ represent conditional expectation and conditional variance based on $X^{\pi}(t)=x$ and $L^{\pi}(t)=l$, respectively. $\bar{\beta}(\epsilon$ $[0,1])$ is the weight of $\bar{L}^{\pi}(t)$, indicating the degree of terminal wealth affected by historical average performance. If we write $\beta=\frac{\bar{\beta}}{\int_{-h}^{0} e^{A u} \mathrm{~d} u}$, then $X^{\pi}(t)+\bar{\beta} \bar{L}^{\pi}(t)=X^{\pi}(t)+\beta L^{\pi}(t)$, which is called the terminal wealth in the sequel. Therefore, we rewrite $(2.4)$ as

$$
\sup _{\pi \in \Pi}\left\{E_{t, x, l}\left[X^{\pi}(T)+\beta L^{\pi}(T)\right]-\frac{\omega}{2} \operatorname{Var}_{t, x, l}\left[X^{\pi}(T)+\beta L^{\pi}(T)\right]\right\} .
$$

In addition, according to Chang et al. [11], the optimal control problem with delay is generally an infinitedimensional problem. To obtain an explicit solution, some additional conditions will be attached. We assume that the value function $V(\cdot)$ is only related to $x$ and $l$. However $L^{\pi}(t)$ is related to $M^{\pi}(t)$. In order to make $V(\cdot)$ only depend on $(t, x, l)$ and then the problem can obtain the explicit expression. Hence we suppose that the following conditions hold:

$$
\gamma_{2}=\beta e^{-A h}, \quad \bar{\gamma}_{1}-A \beta=\left(r-\gamma_{1}-\gamma_{2}+\beta\right) \beta .
$$

Remark 2.1. Note that the two assumptions in (2.6) will be applied over and over below, which is one of the sufficient conditions for optimality when using the maximum principle to solve the control problem with delay (see $[20,22,23])$. In fact, it is also one of the sufficient conditions for finding explicit solutions to stochastic control problems with delay by using the dynamic programming principle. Equation (2.6) can be explained as follows: Firstly, the insurer calculates the integrated delayed wealth $L(t)$ and the pointwise delayed wealth $M(t)$ at the time $t \in[0, T]$ by selecting the average parameter $A$ and the delay parameter $h$. Then, the insurer selects the parameter $\beta$ to determine the weight between $X(t)$ and $L(t)$ in the mean-variance performance measure. Finally, the insurer sets parameters $\gamma_{2}=\beta e^{-A h}$ and $\gamma_{1}=\frac{\beta \int_{-h}^{0} e^{A u} \mathrm{~d} u}{1+\beta \int_{-h}^{0} e^{A u} \mathrm{~d} u}\left(r-\gamma_{2}+\beta+A\right)$ according to (2.6) to determine the weight proportion of past performance $X(t)-\bar{L}(t)$ and $X(t)-M(t)$ at the time $t \in[0, T]$ and adjusts the inflow/outflow of capital accordingly.

The framework corresponding with the problem (2.5) is the traditional investment and reinsurance model, in which the insurer is supposed to be ambiguity-neutral, that is, he/she is assumed to be able to accurately find the probability measure $\mathbb{P}$. However, in reality, the accurate probability measure $\mathbb{P}$ is difficult to find, so most of the insurers are ambiguity-averse, and hope to ensure that the worst-case return can achieve the expected goal. Based on this consideration, we take the uncertainty of probability measure into mean-variance optimization problem (2.5), and model (2.3) under probability measure $\mathbb{P}$ is regarded as the reference model. Since the reference model is skeptical, the insurer will consider some alternative models. Similar to [1], the alternative models are defined as a class of probability measures equivalent to the probability measure $\mathbb{P}$ as follows:

$$
\mathcal{Q}:=\{\mathbb{Q} \mid \mathbb{Q} \sim \mathbb{P}\} .
$$


Definition 2.2 (Admissible strategy). For any fixed $t \in[0, T]$, an investment-reinsurance strategy $\pi(t)=$ $\left\{\left(p_{1}(t), q_{1}(t), q_{2}(t)\right)\right\}$ is said to admissible if it satisfies:

(i) $\pi(t)$ is $\left\{\mathcal{F}_{t}\right\}_{t \in[0, T]}$-progressively measurable;

(ii) $q_{1}(t) \geq 0, q_{2}(t) \geq 0$ and $E_{t, x, l}^{\mathbb{Q}^{*}}\left[\int_{0}^{T}\left(\left(p_{1}(t)\right)^{2}+\left(q_{1}(t)\right)^{2}+\left(q_{2}(t)\right)^{2}\right) \mathrm{d} t\right]<+\infty$, where $\mathbb{Q}^{*}$ is the chosen probability measure to depict the worst case;

(iii) $\forall(t, x, l) \in[0, T] \times \mathbb{R} \times \mathbb{R}$, the $\operatorname{SDDE}(2.3)$ has a pathwise unique solution.

Let $\Pi$ be the set of all admissible strategies.

According to Girsanov's theorem, there exists a progressively measurable process $\varphi(t)=\left(\varphi_{1}(t), \varphi_{2}(t), \varphi_{3}(t)\right)$ for $t \in[0, T]$ such that $\left.\frac{\mathrm{d} \mathbb{Q}}{\mathrm{d} \mathbb{P}}\right|_{\mathcal{F}_{T}}=\Lambda^{\varphi}(T)$, where

$$
\begin{aligned}
\Lambda^{\varphi}(t)= & \exp \left\{-\int_{0}^{t} \varphi_{1}(u) \mathrm{d} W_{0}(u)-\frac{1}{2} \int_{0}^{t} \varphi_{1}^{2}(u) \mathrm{d} u-\int_{0}^{t} \varphi_{2}(u) \mathrm{d} W(u)-\frac{1}{2} \int_{0}^{t} \varphi_{2}^{2}(u) \mathrm{d} u\right. \\
& \left.+\int_{0}^{t} \int_{-1}^{\infty} \ln \varphi_{3}(u) N_{0}\left(\mathrm{~d} u, \mathrm{~d} y_{0}\right)+\int_{0}^{t} \int_{-1}^{\infty}\left(1-\varphi_{3}(u)\right) \nu\left(\mathrm{d} u, \mathrm{~d} y_{0}\right)\right\} .
\end{aligned}
$$

By the definition of $\varphi(t)$, it is easy to show that $\Lambda^{\varphi}(t)$ is a $\mathbb{P}$-martingale under the Novikov's condition, and then $E\left[\Lambda^{\varphi}(t)\right]=1$. By Girsanov's theorem, under the alternative probability measure $\mathbb{Q}$, we have also two standard Brownian motion $\left\{W_{0}^{\mathbb{Q}}(t)\right\}_{t \in[0, T]}$ and $\left\{W^{\mathbb{Q}}(t)\right\}_{t \in[0, T]}$, which are given by

$$
\mathrm{d} W_{0}^{\mathbb{Q}}(t)=\mathrm{d} W_{0}(t)+\varphi_{1}(t) \mathrm{d} t, \quad \mathrm{~d} W^{\mathbb{Q}}(t)=\mathrm{d} W(t)+\varphi_{2}(t) \mathrm{d} t .
$$

And the Poisson process $\left\{N_{0}(t)\right\}_{t \in[0, T]}$ with intensity $\lambda_{0}$ turn into the Poisson process $\left\{N_{0}^{\mathbb{Q}}(t)\right\}_{t \in[0, T]}$ with intensity $\lambda_{0} \varphi_{3}(t)$. We know also that $\left\{W_{0}^{\mathbb{Q}}(t)\right\}_{t \in[0, T]}$ and $\left\{W^{\mathbb{Q}}(t)\right\}_{t \in[0, T]}$ are also mutually independent. In addition, parallel to Branger and Larsen [8], for tractability and ease of interpretation, we suppose that the distribution of jump $Y_{0 i}$ is known and is restricted to be identical under $\mathbb{P}$ and $\mathbb{Q}$. Based on the discussion above, the dynamics of the wealth process (2.3) under alternative probability measure $\mathbb{Q}$ become

$$
\begin{aligned}
\mathrm{d} X^{\pi}(t)= & {\left[\left(r-\gamma_{1}-\gamma_{2}\right) X^{\pi}(t)+\bar{\gamma}_{1} L^{\pi}(t)+\gamma_{2} M^{\pi}(t)+(\alpha-r) p_{1}(t)\right.} \\
& +\left(\theta_{1}-\eta_{1}+q_{1}(t) \eta_{1}\right) a_{1}+\left(\theta_{2}-\eta_{2}+q_{2}(t) \eta_{2}\right) a_{2}-\sigma p_{1}(t) \varphi_{2}(t) \\
& \left.-\sqrt{b_{1}^{2} q_{1}^{2}(t)+b_{2}^{2} q_{2}^{2}(t)+2 q_{1}(t) q_{2}(t) \lambda \mu_{11} \mu_{21}} \varphi_{1}(t)\right] \mathrm{d} t \\
& +\sqrt{b_{1}^{2} q_{1}^{2}(t)+b_{2}^{2} q_{2}^{2}(t)+2 q_{1}(t) q_{2}(t) \lambda \mu_{11} \mu_{21}} \mathrm{~d} W_{0}^{\mathbb{Q}}(t) \\
& +p_{1}(t) \sigma \mathrm{d} W^{\mathbb{Q}}(t)+\int_{-1}^{\infty} p_{1}(t) y_{0} N_{0}^{\mathbb{Q}}\left(\mathrm{d} t, \mathrm{~d} y_{0}\right) .
\end{aligned}
$$

In what follows, we consider the robust mean-variance optimization problem. As Maenhout [18], Yi et al. [25] and Zeng et al. [28] mentioned, we formulate the following robust optimization problem to modify the mean-variance problem (2.5)

$$
\sup _{\pi \in \Pi} J(t, x, l ; \pi)=\sup _{\pi \Pi} \inf _{\mathbb{Q} \in \mathcal{Q}} \hat{J}(t, x, l ; \pi, \mathbb{Q}) .
$$

Here

$$
\hat{J}(t, x, l ; \pi, \mathbb{Q})=E_{t, x, l}^{\mathbb{Q}}\left[X^{\pi}(T)+\beta L^{\pi}(T)\right]-\frac{\omega}{2} \operatorname{Var}_{t, x, l}^{\mathbb{Q}}\left[X^{\pi}(T)+\beta L^{\pi}(T)\right]
$$




$$
+E_{t, x, l}^{\mathbb{Q}}\left[\int_{t}^{T}\left(\frac{\varphi_{1}^{2}(u)}{2 \phi_{1}(u)}+\frac{\varphi_{2}^{2}(u)}{2 \phi_{2}(u)}+\frac{\lambda_{0}\left(\varphi_{3}(u) \ln \varphi_{3}(u)-\varphi_{3}(u)+1\right)}{\phi_{3}(u)}\right) \mathrm{d} u\right],
$$

where $\phi_{1}(t), \phi_{2}(t)$ and $\phi_{3}(t)$ are all nonnegative and are used to capture the AAI's ambiguity aversions. And the larger $\phi_{1}(t), \phi_{2}(t)$ and $\phi_{3}(t)$, the more ambiguity-averse the AAI. Furthermore, the deviations from the reference measure $\mathbb{P}$ are penalized by the first three terms in the above expression. Similar to Branger and Larsen [8], the deviations depend on the relative entropy arising from the jump-diffusion risk, in which the increase in relative entropy from $t$ to $t+\mathrm{d} t$ is given by

$$
\left[\frac{1}{2}\left(\varphi_{1}^{2}(t)+\varphi_{2}^{2}(t)\right)+\lambda_{0}\left(\varphi_{3}(t) \ln \varphi_{3}(t)-\varphi_{3}(t)+1\right)\right] \mathrm{d} t .
$$

The detailed derivation of the above formula is shown in Appendix A. Since there exists a non-linear function of the expectation of terminal wealth in the objective functional (2.9), the optimization problem is timeinconsistent. Most literature solves the mean-variance problem by setting a precommitment, in which optimal strategies obtained are time-inconsistent. In fact, time-consistency cannot be neglected for a rational decisionmaker who aims to seek an equilibrium strategy that is optimal at a time and still be optimal as time goes forward into the future. Hence, we shall define the following time-consistent equilibrium strategy according to Björk and Murgoci [6] and Björk et al. [7].

Definition 2.3. Consider an admissible strategy $\pi^{*}$, which can be informally viewed as a candidate equilibrium strategy. And choose arbitrarily a fixed $\pi \in \Pi$, a real number $\varepsilon(>0)$ and a given initial point $(t, x, l) \in[0, T] \times$ $\mathbb{R} \times \mathbb{R}$, define the strategy $\pi_{\varepsilon}$ as follows:

$$
\pi_{\varepsilon}= \begin{cases}\hat{\pi}(u, x, l), & (u, x, l) \in[t, t+\varepsilon) \times \mathbb{R} \times \mathbb{R}, \\ \pi^{*}(u, x, l), & (u, x, l) \in[t+\varepsilon, T] \times \mathbb{R} \times \mathbb{R} .\end{cases}
$$

If

$$
\liminf _{\varepsilon \downarrow 0} \frac{J\left(t, x, l ; \pi^{*}\right)-J\left(t, x, l ; \pi_{\varepsilon}\right)}{\varepsilon} \geq 0,
$$

we call that $\pi^{*}$ is an equilibrium strategy and the equilibrium value function $W(t, x, l)$ is given by

$$
W(t, x, l)=J\left(t, x, l ; \pi^{*}\right) .
$$

According to Definition 2.3, the equilibrium strategy above is time-consistent. We aim to seek an equilibrium strategy $\pi^{*}$ and the corresponding equilibrium value function. To give the extended HJB equation and Verification Theorem conveniently, we define a variational operator. Let $\mathcal{C}^{1,2,1}([0, T] \times \mathbb{R} \times \mathbb{R})$ denotes a space of any function $\psi(t, x, l)$ which $\psi(t, x, l)$ itself and its derivatives $\psi_{t}(t, x, l), \psi_{x}(, t, x, l), \psi_{x x}(t, x, l)$, and $\psi_{l}(t, x, l)$ are continuous on $[0, T] \times \mathbb{R} \times \mathbb{R} \times \mathbb{R} \times \mathbb{R}$. For any function $\psi(t, x, l) \in \mathcal{C}^{1,2,1}([0, T] \times \mathbb{R} \times \mathbb{R})$ and a given $\pi \in \Pi$, the variational operator corresponding to the alternative measure $\mathbb{Q}$ is defined as follows:

$$
\begin{aligned}
\mathcal{L}^{\pi} \psi(t, x, l)= & \psi_{t}+\left[\left(r-\gamma_{1}-\gamma_{2}\right) x+\bar{\gamma}_{1} l+\gamma_{2} m+(\alpha-r) p_{1}+\left(\theta_{1}-\eta_{1}+q_{1} \eta_{1}\right) a_{1}\right. \\
& \left.+\left(\theta_{2}-\eta_{2}+q_{2} \eta_{2}\right) a_{2}-\sqrt{b_{1}^{2} q_{1}^{2}(t)+b_{2}^{2} q_{2}^{2}(t)+2 q_{1} q_{2} \lambda \mu_{11} \mu_{21}} \varphi_{1}-\sigma p_{1} \varphi_{2}\right] \psi_{x} \\
& +\left(x-A l-e^{-A h} m\right) \psi_{l}+\frac{1}{2}\left(b_{1}^{2} q_{1}^{2}+b_{2}^{2} q_{2}^{2}+2 q_{1} q_{2} \lambda \mu_{11} \mu_{21}+p_{1}^{2} \sigma^{2}\right) \psi_{x x} \\
& +\lambda_{0} \varphi_{3} E^{\mathbb{Q}}\left[\psi\left(t, x+p_{1} y_{0}, l\right)-\psi(t, x, l)\right] .
\end{aligned}
$$

Before finding the robust equilibrium strategy, the following theorem gives the verifications for the extended HJB equation corresponding to the problem (2.9). 
Theorem 2.4 (Verification theorem). For the robust mean-variance problem (2.9), we assume that there exist two real-valued functions $V(t, x, l), g(t, x, l) \in \mathcal{C}^{1,2,1}([0, T] \times \mathbb{R} \times \mathbb{R})$ satisfying the following conditions:

$$
\begin{gathered}
\left\{\begin{array}{l}
\sup _{\pi \in \Pi} \inf _{\mathbb{Q} \in \mathcal{Q}}\left\{\mathcal{L}^{\pi, \varphi} V(t, x, l)-\frac{\omega}{2} \mathcal{L}^{\pi, \varphi} g^{2}(t, x, l)+\omega g(t, x, l) \mathcal{L}^{\pi, \varphi} g(t, x, l)+\frac{\varphi_{1}^{2}(t)}{2 \phi_{1}(t)}\right. \\
\left.\quad+\frac{\varphi_{2}^{2}(t)}{2 \phi_{2}(t)}+\frac{\lambda_{0}\left(\varphi_{3}(t) \ln \varphi_{3}(t)-\varphi_{3}(t)+1\right)}{\phi_{3}(t)}\right\}=0, \\
\mathcal{L}^{\pi^{*}, \varphi^{*}} g(t, x, l)=0,
\end{array}\right. \\
\left(\pi^{*}, \varphi^{*}\right):=\arg \sup _{\pi \in \Pi} \inf _{\mathbb{Q} \in \mathcal{Q}}\left\{\mathcal{L}^{\pi, \varphi} V(t, x, l)-\frac{\omega}{2} \mathcal{L}^{\pi, \varphi} g^{2}(t, x, l)+\omega g(t, x, l) \mathcal{L}^{\pi, \varphi} g(t, x, l)\right. \\
\left.\quad+\frac{\varphi_{1}^{2}(t)}{2 \phi_{1}(t)}+\frac{\varphi_{2}^{2}(t)}{2 \phi_{2}(t)}+\frac{\lambda_{0}\left(\varphi_{3}(t) \ln \varphi_{3}(t)-\varphi_{3}(t)+1\right)}{\phi_{3}(t)}\right\}, \\
V(T, x, l)=x+\beta l, \quad g(T, x, l)=x+\beta l,
\end{gathered}
$$

then $E_{t, x, l}^{\mathbb{Q}}\left[X^{\pi^{*}}(T)+\beta L^{\pi^{*}}(T)\right]=g(t, x, l), W(t, x, l)=V(t, x, l)$ and $\pi^{*}$ is the robust equilibrium investmentreinsurance strategy.

The proof for the verification theorem is similar to the Theorem 5.2 in Björk et al. [7], hence here is omitted.

\section{The SOlution to The Optimization PROBlem}

In this section, we derive the explicit solution to the robust equilibrium strategy and the corresponding robust equilibrium value function for investment-reinsurance problem (2.9). As Branger and Larsen [8] and Chen and Yang [12] mentioned, to make the problem (2.9) tractable and ensure that the penalty in problem (2.9) is reasonable, some restrictions must be imposed on the ambiguity-aversion parameter. We suppose that $\phi_{1}(t)=\xi_{1}, \phi_{2}(t)=\xi_{2}$ and $\phi_{3}(t)=\xi_{3}$, where $\xi_{1}, \xi_{2}$ and $\xi_{3}$ are all nonnegative. Then according to the variational operator (2.12), (2.13) can be rewritten as

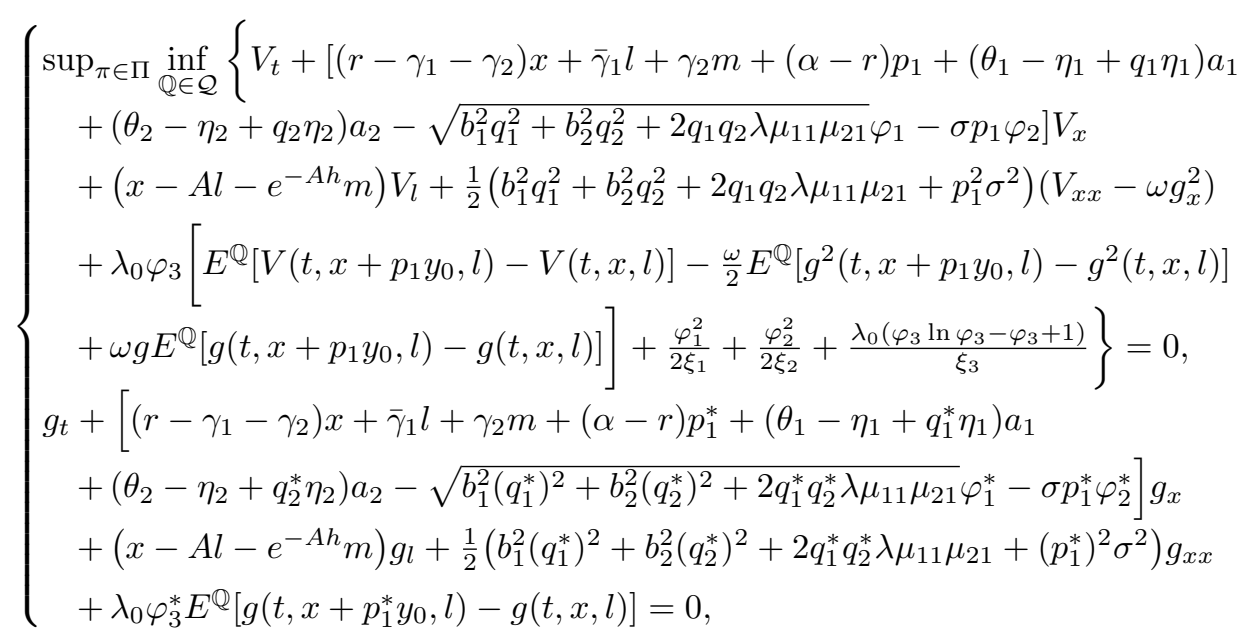

where the terminal value conditions are given by $V(T, x, l)=x+\beta l$ and $g(T, x, l)=x+\beta l$.

To guarantee the insurance retention $q_{1}(\cdot)$ and $q_{2}(\cdot)$ are non-negative, we give the following lemma.

Lemma 3.1. The parameters $\lambda, \lambda_{1}, \lambda_{2}, \mu_{11}, \mu_{21}, \mu_{12}$ and $\mu_{22}$ given in Section 2 above satisfy the following inequalities:

$$
b_{1}^{2} b_{2}^{2}>\lambda^{2} \mu_{11}^{2} \mu_{21}^{2}, \quad \frac{\lambda \mu_{11} \mu_{21}}{b_{2}^{2}} \frac{a_{2}}{a_{1}}<1<\frac{b_{1}^{2}}{\lambda \mu_{11} \mu_{21}} \frac{a_{2}}{a_{1}} .
$$


Proof. See Appendix B.

Considering $q_{1}(\cdot)$ and $q_{2}(\cdot)$ are non-negative, according to Lemma 3.1, the following three cases need to be discussed.

Case 1. For $\eta_{1} \leq \frac{\lambda \mu_{11} \mu_{21}}{b_{2}^{2}} \frac{a_{2}}{a_{1}} \eta_{2}$, we have $n_{1} \leq 0$ and $n_{2}>0$;

Case 2. For $\frac{\lambda \mu_{11} \mu_{21}}{b_{2}^{2}} \frac{a_{2}}{a_{1}} \eta_{2}<\eta_{1}<\frac{b_{1}^{2}}{\lambda \mu_{11} \mu_{21}} \frac{a_{2}}{a_{1}} \eta_{2}$, we have $n_{1}>0$ and $n_{2}>0$;

Case 3. For $\eta_{1} \geq \frac{b_{1}^{2}}{\lambda \mu_{11} \mu_{21}} \frac{a_{2}}{a_{1}} \eta_{2}$, we have $n_{1}>0$ and $n_{2} \leq 0$.

Here $n_{1}=\frac{a_{1} \eta_{1} b_{2}^{2}-a_{2} \eta_{2} \lambda \mu_{11} \mu_{21}}{b_{1}^{2} b_{2}^{2}-\lambda^{2} \mu_{11}^{2} \mu_{21}^{2}}$ and $n_{2}=\frac{a_{2} \eta_{2} b_{1}^{2}-a_{1} \eta_{1} \lambda \mu_{11} \mu_{21}}{b_{1}^{2} b_{2}^{2}-\lambda^{2} \mu_{11}^{2} \mu_{21}^{2}}$. We only detail analyze Case 2 in the following theorem. The other two cases can be similarly deduced.

Next, we give the explicit expression to the robust equilibrium strategy and the corresponding robust equilibrium value function by the following theorem.

Theorem 3.2. For the mean-variance problem (2.9) under Case 2, the robust equilibrium investment and reinsurance strategy are given by

$$
\begin{aligned}
p_{1}^{*}(t)= & \frac{e^{-\left(r-\gamma_{1}-\gamma_{2}+\beta\right)(T-t)}}{\omega+\xi_{2}}\left[\frac{\alpha-r}{\sigma^{2}}-\frac{\lambda_{0}\left(\omega \mu_{02} p_{1}^{*}(t) e^{\left(r-\gamma_{1}-\gamma_{2}+\beta\right)(T-t)}-\mu_{01}\right)}{\sigma^{2}}\right. \\
& \left.\times e^{\xi_{3}\left(\frac{\omega}{2} \mu_{02}\left(p_{1}^{*}(t)\right)^{2} e^{2\left(r-\gamma_{1}-\gamma_{2}+\beta\right)(T-t)}-\mu_{01} p_{1}^{*}(t) e^{\left(r-\gamma_{1}-\gamma_{2}+\beta\right)(T-t)}\right)}\right], \\
q_{1}^{*}(t)= & \frac{n_{1}}{\omega+\xi_{1}} e^{-\left(r-\gamma_{1}-\gamma_{2}+\beta\right)(T-t)}, \\
q_{2}^{*}(t)= & \frac{n_{2}}{\omega+\xi_{1}} e^{-\left(r-\gamma_{1}-\gamma_{2}+\beta\right)(T-t)},
\end{aligned}
$$

and the corresponding robust equilibrium value function is

$$
\begin{aligned}
V(t, x, l)= & e^{\left(r-\gamma_{1}-\gamma_{2}+\beta\right)(T-t)}(x+\beta l)-\frac{\left[\left(\theta_{1}-\eta_{1}\right) a_{1}+\left(\theta_{2}-\eta_{2}\right) a_{2}\right]}{r-\gamma_{1}-\gamma_{2}+\beta}\left(1-e^{\left(r-\gamma_{1}-\gamma_{2}+\beta\right)(T-t)}\right) \\
& +\frac{1}{\omega+\xi_{1}}\left(a_{1} n_{1} \eta_{1}+a_{2} n_{2} \eta_{2}-\frac{1}{2} b_{1}^{2} n_{1}^{2}-\frac{1}{2} b_{2}^{2} n_{2}^{2}-\lambda \mu_{11} \mu_{21} n_{1} n_{2}\right)(T-t) \\
& +\int_{t}^{T}\left[(\alpha-r) p_{1}^{*}(s) e^{\left(r-\gamma_{1}-\gamma_{2}+\beta\right)(T-s)}\right. \\
& -\frac{\left(p_{1}^{*}(s)\right)^{2} \sigma^{2}\left(\omega+\xi_{2}\right) e^{2\left(r-\gamma_{1}-\gamma_{2}+\beta\right)(T-s)}}{2} \\
& \left.+\frac{\lambda_{0}}{\xi_{3}}\left(1-e^{\xi_{3}\left(\frac{\omega}{2} \mu_{02}\left(p_{1}^{*}(s)\right)^{2} e^{2\left(r-\gamma_{1}-\gamma_{2}+\beta\right)(T-s)}-\mu_{01} p_{1}^{*}(s) e^{\left(r-\gamma_{1}-\gamma_{2}+\beta\right)(T-s)}\right)}\right)\right] \mathrm{d} s .
\end{aligned}
$$

Besides, the worst-case measure is as follows:

$$
\begin{aligned}
& \varphi_{1}^{*}(t)=\xi_{1} \sqrt{b_{1}^{2}\left(q_{1}^{*}(t)\right)^{2}+b_{2}^{2}\left(q_{2}^{*}(t)\right)^{2}+2 q_{1}^{*}(t) q_{2}^{*}(t) \lambda \mu_{11} \mu_{21}} e^{\left(r-\gamma_{1}-\gamma_{2}+\beta\right)(T-t)}, \\
& \varphi_{2}^{*}(t)=\xi_{2} \sigma p_{1}^{*}(t) e^{\left(r-\gamma_{1}-\gamma_{2}+\beta\right)(T-t)} \\
& \varphi_{3}^{*}(t)=e^{\xi_{3}\left(\frac{\omega}{2} \mu_{02}\left(p_{1}^{*}(t)\right)^{2} e^{2\left(r-\gamma_{1}-\gamma_{2}+\beta\right)(T-t)}-\mu_{01} p_{1}^{*}(t) e^{\left(r-\gamma_{1}-\gamma_{2}+\beta\right)(T-t)}\right) .}
\end{aligned}
$$

Proof. See Appendix C.

Proposition 3.3. Equation (3.3) has a unique positive root, that is, there exists a unique $p_{1}^{*}(t) \in[0,+\infty)$ that satisfies equation (3.3). 
Proof. See Appendix D.

In what follows, we present some special cases of our model. If the insurer is ambiguity-neutral, then the ambiguity-aversion coefficients $\xi_{1}=\xi_{2}=\xi_{3}=0$, and then $\varphi_{1}(t)=\varphi_{2}(t)=0$ and $\varphi_{3}(t)=1$. Further, the wealth process and the optimization problem for the ambiguity-neutral insurer are given by (2.3) and (2.5), respectively. And the extended HJB equation corresponding to problem (2.5) is derived by

$$
\left\{\begin{aligned}
\sup _{\pi \in \Pi}\left\{V_{t}+\left[\left(r-\gamma_{1}-\gamma_{2}\right) x+\bar{\gamma}_{1} l+\gamma_{2} m+(\alpha-r) p_{1}+\left(\theta_{1}-\eta_{1}+q_{1} \eta_{1}\right) a_{1}\right.\right. \\
\left.\quad+\left(\theta_{2}-\eta_{2}+q_{2} \eta_{2}\right) a_{2}\right] V_{x}+\left(x-A l-e^{-A h} m\right) V_{l}+\frac{1}{2}\left(b_{1}^{2} q_{1}^{2}+b_{2}^{2} q_{2}^{2}+2 q_{1} q_{2} \lambda \mu_{11} \mu_{21}\right. \\
\left.\quad+p_{1}^{2} \sigma^{2}\right)\left(V_{x x}-\omega g_{x}^{2}\right)+\lambda_{0}\left[E\left[V\left(t, x+p_{1} y_{0}, l\right)-V(t, x, l)\right]\right. \\
\left.\left.\quad-\frac{\omega}{2} E\left[g^{2}\left(t, x+p_{1} y_{0}, l\right)-g^{2}(t, x, l)\right]+\omega g E\left[g\left(t, x+p_{1} y_{0}, l\right)-g(t, x, l)\right]\right]\right\}=0, \\
g_{t}+\left[\left(r-\gamma_{1}-\gamma_{2}\right) x+\bar{\gamma}_{1} l+\gamma_{2} m+(\alpha-r) p_{1}^{*}+\left(\theta_{1}-\eta_{1}+q_{1}^{*} \eta_{1}\right) a_{1}+\left(\theta_{2}-\eta_{2}\right.\right. \\
\left.\left.\quad+q_{2}^{*} \eta_{2}\right) a_{2}\right] g_{x}+\left(x-A l-e^{-A h} m\right) g_{l}+\frac{1}{2}\left(b_{1}^{2}\left(q_{1}^{*}\right)^{2}+b_{2}^{2}\left(q_{2}^{*}\right)^{2}+2 q_{1}^{*} q_{2}^{*} \lambda \mu_{11} \mu_{21}\right. \\
\left.\quad+\left(p_{1}^{*}\right)^{2} \sigma^{2}\right) g_{x x}+\lambda_{0} E\left[g\left(t, x+p_{1}^{*} y_{0}, l\right)-g(t, x, l)\right]=0 .
\end{aligned}\right.
$$

Similar to the derivation of theorem 3.2, we present the following corollary.

Corollary 3.4. Under Case 2, for the ANI in our model, the equilibrium investment and reinsurance strategy are given by:

$$
\begin{aligned}
\hat{p}_{1}^{*}(t) & =\frac{\alpha-r+\lambda_{0} \mu_{01}}{\omega\left(\sigma^{2}+\lambda_{0} \mu_{02}\right)} e^{-\left(r-\gamma_{1}-\gamma_{2}+\beta\right)(T-t)}, \\
\hat{q}_{1}^{*}(t) & =\frac{n_{1}}{\omega} e^{-\left(r-\gamma_{1}-\gamma_{2}+\beta\right)(T-t)}, \\
\hat{q}_{2}^{*}(t) & =\frac{n_{2}}{\omega} e^{-\left(r-\gamma_{1}-\gamma_{2}+\beta\right)(T-t)},
\end{aligned}
$$

and the corresponding equilibrium value function is

$$
\begin{aligned}
\hat{V}(t, x, l)= & e^{\left(r-\gamma_{1}-\gamma_{2}+\beta\right)(T-t)}(x+\beta l)-\frac{\left[\left(\theta_{1}-\eta_{1}\right) a_{1}+\left(\theta_{2}-\eta_{2}\right) a_{2}\right]}{r-\gamma_{1}-\gamma_{2}+\beta} \\
& \times\left(1-e^{\left(r-\gamma_{1}-\gamma_{2}+\beta\right)(T-t)}\right)+\frac{\left(\alpha-r+\lambda_{0} \mu_{01}\right)^{2}}{2 \omega\left(\sigma^{2}+\lambda_{0} \mu_{02}\right)}(T-t) \\
& +\frac{1}{\omega}\left(a_{1} n_{1} \eta_{1}+a_{2} n_{2} \eta_{2}-\frac{b_{1}^{2} n_{1}^{2}}{2}-\frac{b_{2}^{2} n_{2}^{2}}{2}-\lambda \mu_{11} \mu_{21} n_{1} n_{2}\right)(T-t) .
\end{aligned}
$$

Next, the utility loss is investigated under the case of ignoring model ambiguity. The optimal strategy for ANI is the suboptimal strategy of AAI, hence the strategy given in Corollary 3.4 is called the suboptimal strategy. Let $\hat{\pi}^{*}=\left(\hat{p}_{1}^{*}, \hat{q}_{1}^{*}, \hat{q}_{2}^{*}\right)$, and assume that the AAI takes the suboptimal strategy $\hat{\pi}^{*}$ given in Corollary 3.4 , then the corresponding value function is

$$
V_{\mathrm{sub}}(t, x, l)=\inf _{\mathbb{Q} \in \mathcal{Q}} J\left(t, x, l ; \hat{\pi}^{*}\right) .
$$

The following proposition presents directly the value function under the strategy $\hat{\pi}^{*}$, which the calculation process is similar to Theorem 3.2 .

Proposition 3.5. For problem (3.14) under Case 2, the value function is given by

$$
V_{\mathrm{sub}}(t, x, l)=e^{\left(r-\gamma_{1}-\gamma_{2}+\beta\right)(T-t)}(x+\beta l)-\frac{\left[\left(\theta_{1}-\eta_{1}\right) a_{1}+\left(\theta_{2}-\eta_{2}\right) a_{2}\right]}{r-\gamma_{1}-\gamma_{2}+\beta}
$$




$$
\begin{aligned}
& \times\left(1-e^{\left(r-\gamma_{1}-\gamma_{2}+\beta\right)(T-t)}\right)+\frac{\left(a_{1} \eta_{1} n_{1}+a_{2} \eta_{2} n_{2}\right)(T-t)}{\omega} \\
& -\frac{\omega+\xi_{1}}{2 \omega^{2}}\left(b_{1}^{2} n_{1}^{2}+b_{2}^{2} n_{2}^{2}+2 \lambda \mu_{11} \mu_{21} n_{1} n_{2}\right)(T-t) \\
& +\frac{(\alpha-r)\left(\alpha-r+\lambda_{0} \mu_{01}\right)(T-t)}{\omega\left(\sigma^{2}+\lambda_{0} \mu_{02}\right)}-\frac{\sigma^{2}\left(\alpha-r+\lambda_{0} \mu_{01}\right)^{2}\left(\xi_{2}+\omega\right)(T-t)}{2 \omega^{2}\left(\sigma^{2}+\lambda_{0} \mu_{02}\right)^{2}} \\
& +\frac{\lambda_{0}}{\xi_{3}}\left(1-e^{\xi_{3}\left(\frac{\mu_{02}\left(\alpha-r+\lambda_{0} \mu_{01}\right)^{2}}{2 \omega\left(\sigma^{2}+\lambda_{0} \mu_{02}\right)^{2}}-\frac{\mu_{01}\left(\alpha-r+\lambda_{0} \mu_{01}\right)}{\omega\left(\sigma^{2}+\lambda_{0} \mu_{02}\right)}\right)}\right)(T-t) .
\end{aligned}
$$

Furthermore, we define the following utility loss function:

$$
\mathrm{UL}:=1-\frac{V_{\mathrm{sub}}(t, x, l)}{V(t, x, l)} .
$$

Remark 3.6. From Corollary 3.4, if we do not consider the price jump of risky assets, then the investment strategy becomes $\hat{p}_{1}^{*}(t)=\frac{\alpha-r}{\omega \sigma^{2}}$, the reinsurance strategy is not affected, and the corresponding value function becomes

$$
\begin{aligned}
V_{0}(t, x, l)= & e^{\left(r-\gamma_{1}-\gamma_{2}+\beta\right)(T-t)}(x+\beta l)-\frac{\left[\left(\theta_{1}-\eta_{1}\right) a_{1}+\left(\theta_{2}-\eta_{2}\right) a_{2}\right]}{r-\gamma_{1}-\gamma_{2}+\beta} \\
& \times\left(1-e^{\left(r-\gamma_{1}-\gamma_{2}+\beta\right)(T-t)}\right)+\frac{(\alpha-r)^{2}}{2 \omega \sigma^{2}}(T-t) \\
& +\frac{1}{\omega}\left(a_{1} n_{1} \eta_{1}+a_{2} n_{2} \eta_{2}-\frac{b_{1}^{2} n_{1}^{2}}{2}-\frac{b_{2}^{2} n_{2}^{2}}{2}-\lambda \mu_{11} \mu_{21} n_{1} n_{2}\right)(T-t) .
\end{aligned}
$$

According to (2.6) and the discussion in Remark 2.1, $A=h=\beta=0$ when the delay is without consideration. Hence by Theorem 3.2, we give the following corollary.

Corollary 3.7. Under Case 2 and no-delay case, the robust equilibrium investment and reinsurance strategy are given by

$$
\begin{aligned}
& \bar{p}_{1}^{*}(t)=\frac{e^{-r(T-t)}}{\omega+\xi_{2}}\left[\frac{\alpha-r}{\sigma^{2}}-\frac{\lambda_{0}\left(\omega \mu_{02} \bar{p}_{1}^{*} e^{r(T-t)}-\mu_{01}\right)}{\sigma^{2}} \times e^{\left.\xi_{3}\left(\frac{\omega}{2} \mu_{02}\left(\bar{p}_{1}^{*}\right)^{2} e^{2 r(T-t)}-\mu_{01} \bar{p}_{1}^{*} e^{r(T-t)}\right)\right],}\right. \\
& \bar{q}_{1}^{*}(t)=\frac{n_{1}}{\omega+\xi_{1}} e^{-r(T-t)}, \\
& \bar{q}_{2}^{*}(t)=\frac{n_{2}}{\omega+\xi_{1}} e^{-r(T-t)},
\end{aligned}
$$

and the corresponding equilibrium value function is

$$
\begin{aligned}
\bar{V}(t, x, l)= & e^{r(T-t)}(x+\beta l)-\frac{\left[\left(\theta_{1}-\eta_{1}\right) a_{1}+\left(\theta_{2}-\eta_{2}\right) a_{2}\right]}{r}\left(1-e^{r(T-t)}\right) \\
& +\frac{1}{\omega+\xi_{1}}\left(a_{1} n_{1} \eta_{1}+a_{2} n_{2} \eta_{2}-\frac{1}{2} b_{1}^{2} n_{1}^{2}-\frac{1}{2} b_{2}^{2} n_{2}^{2}-\lambda \mu_{11} \mu_{21} n_{1} n_{2}\right)(T-t) \\
& +\int_{t}^{T}\left[(\alpha-r) \bar{p}_{1}^{*}(s) e^{r(T-s)}-\frac{\left(\bar{p}_{1}^{*}(s)\right)^{2} \sigma^{2}\left(\omega+\xi_{2}\right) e^{2 r(T-s)}}{2}\right. \\
& +\frac{\lambda_{0}}{\xi_{3}}\left(1-e^{\left.\left.\xi_{3}\left(\frac{\omega}{2} \mu_{02}\left(\bar{p}_{1}^{*}(s)\right)^{2} e^{2 r(T-s)}-\mu_{01} \bar{p}_{1}^{*}(s) e^{r(T-s)}\right)\right)\right] \mathrm{d} s .}\right.
\end{aligned}
$$


Remark 3.8. Based on Corollary 3.7, we further ignore the dependence of insurance business, that is, $\lambda=0$, then the robust equilibrium investment and reinsurance strategy are given by

$$
\begin{aligned}
\bar{p}_{01}^{*}(t) & =\frac{e^{-r(T-t)}}{\omega+\xi_{2}}\left[\frac{\alpha-r}{\sigma^{2}}-\frac{\lambda_{0}\left(\omega \mu_{02} \bar{p}_{1}^{*} e^{r(T-t)}-\mu_{01}\right)}{\sigma^{2}} \times e^{\left.\xi_{3}\left(\frac{\omega}{2} \mu_{02}\left(\bar{p}_{1}^{*}\right)^{2} e^{2 r(T-t)}-\mu_{01} \bar{p}_{1}^{*} e^{r(T-t)}\right)\right],}\right. \\
\bar{q}_{01}^{*}(t) & =\frac{\mu_{11} \eta_{1}}{\mu_{12}\left(\omega+\xi_{1}\right)} e^{-r(T-t)}, \\
\bar{q}_{02}^{*}(t) & =\frac{\mu_{21} \eta_{2}}{\mu_{22}\left(\omega+\xi_{1}\right)} e^{-r(T-t)},
\end{aligned}
$$

and the corresponding equilibrium value function is

$$
\begin{aligned}
\bar{V}(t, x)= & e^{r(T-t)}(x+\beta l)-\frac{\left[\left(\theta_{1}-\eta_{1}\right) a_{1}+\left(\theta_{2}-\eta_{2}\right) a_{2}\right]}{r}\left(1-e^{r(T-t)}\right) \\
& +\frac{1}{2\left(\omega+\xi_{1}\right)}\left(\frac{\lambda_{1} \mu_{11}^{2} \eta_{1}^{2}}{\mu_{12}}+\frac{\lambda_{2} \mu_{21}^{2} \eta_{2}^{2}}{\mu_{22}}\right)(T-t) \\
& +\int_{t}^{T}\left[(\alpha-r) \bar{p}_{1}^{*}(s) e^{r(T-s)}-\frac{\left(\bar{p}_{1}^{*}(s)\right)^{2} \sigma^{2}\left(\omega+\xi_{2}\right) e^{2 r(T-s)}}{2}\right. \\
& \left.+\frac{\lambda_{0}}{\xi_{3}}\left(1-e^{\xi_{3}\left(\frac{\omega}{2} \mu_{02}\left(\bar{p}_{1}^{*}(s)\right)^{2} e^{2 r(T-s)}-\mu_{01} \bar{p}_{1}^{*}(s) e^{r(T-s)}\right)}\right)\right] \mathrm{d} s .
\end{aligned}
$$

From the above discussion, we can find that $\bar{p}_{01}^{*}(t), \bar{q}_{01}^{*}(t)$ and $\bar{q}_{02}^{*}(t)$ are consistent with $\pi_{2}^{*}(t)$ and $p_{2}^{*}(t)$ in Zeng et al. [28] when the insurance business are independent of each other. The difference is that the value function $\bar{V}(t, x)$ is larger than that $V_{2}(t, x)$ given in Zeng et al. [28] due to the increase of insurance business lines in this paper.

\section{SEnSitivity ANALYSIS}

This section presents a numerical example to illustrate the effects of some model parameters on the robust equilibrium investment and reinsurance strategy and utility losses by ignoring model uncertainty, in which we give some economic explanations. According to the model settings in Section 2, unless otherwise stated, we select the following parameters throughout this section: $\theta_{1}=\theta_{2}=0.2, \eta_{1}=\eta_{2}=0.4, \mu_{11}=\mu_{21}=0.2$, $\mu_{12}=\mu_{22}=0.3, \lambda=1, \lambda_{1}=2, \lambda_{2}=3, \alpha=0.08, r=0.03, \sigma=0.25, \lambda_{0}=1, \mu_{01}=1, \mu_{02}=2, \xi_{1}=\xi_{2}=0.5$, $\xi_{3}=0.7, \omega=0.3, x=1, t=0, T=3, A=0.1, \beta=0.1$ and $h=1$.

Figure 1 depicts the effect of parameters $A, h$ and $\beta$ on the robust equilibrium investment and reinsurance strategy. As shown in Figure 1a, the robust equilibrium investment strategy $p_{1}^{*}(t)$ invested in the risky asset decreases with $A$. According to the definition of the average delayed wealth $\bar{L}(t)$, we can find that the larger is $A$, the smaller is the proportion of earlier wealth taken up in the construction of average delayed wealth $\bar{L}(t)$. That is to say, the more the insurer pays attention to the wealth close to the current time, which is a kind of short-sighted behavior. In practice, investors' shortsightedness often increases the risk, and they may take risks for short-term interests. Thus, when $A$ is larger, the insurer will reduce the amount invested in risky assets to control the overall risk. In the same mechanism as the effect of $A$ on $p_{1}^{*}(t)$, from Figures $1 \mathrm{~b}$ and $1 \mathrm{c}$, we can also see that retention ratio $q_{1}^{*}(t)$ and $q_{2}^{*}(t)$ also decrease with $A$. Figure $1 \mathrm{~d}$ shows the impact of $h$ on the robust equilibrium investment strategy. Obviously, we can see that the amount invested in risky assets increases with the increase of $h$. Intuitively, with an increase of $h$, the horizontal range of the average becomes longer, and the average delayed wealth is more stable. That is, with an increase of $h$, the insurer's ability to control risk will increase, so the amount invested in risky assets will increase. Similarly, as depicted in Figures 1e and 1f, retention $q_{1}^{*}(t)$ and $q_{2}^{*}(t)$ increase with $h$. Figure $1 \mathrm{~g}$ shows that with $\beta$ increases, the average delayed wealth $\bar{L}(t)$ takes up a larger weight in the terminal wealth. So the averaging effect should definitely reduce the overall risk of the insurer's terminal wealth $X(t)+\beta \bar{L}(t)$. That is, to achieve the same level of the expected terminal 


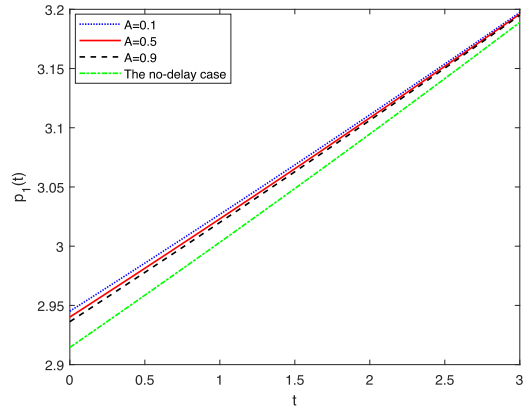

(a)

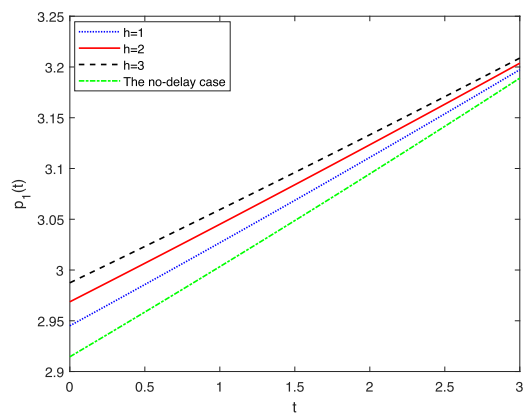

(d)

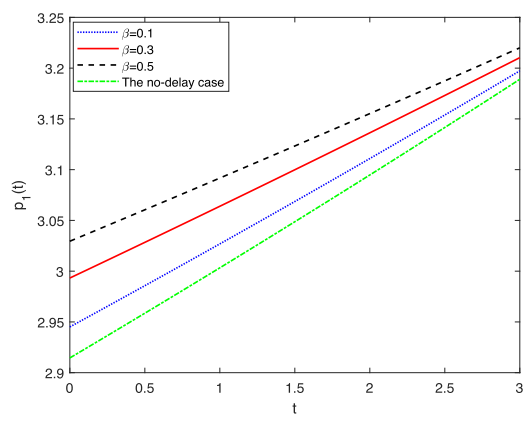

(g)

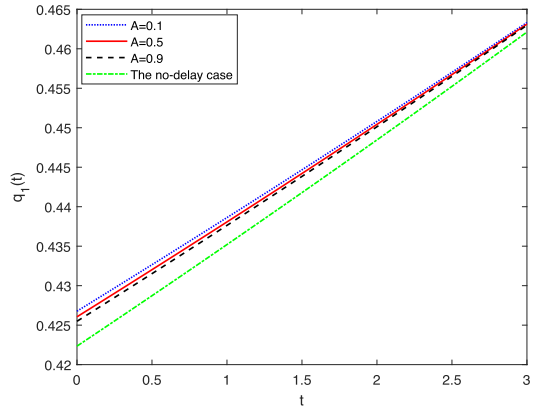

(b)

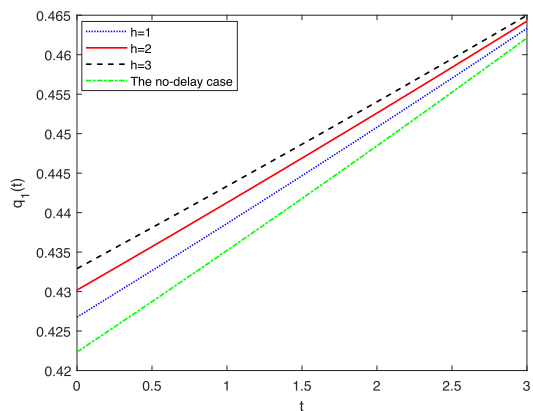

(e)

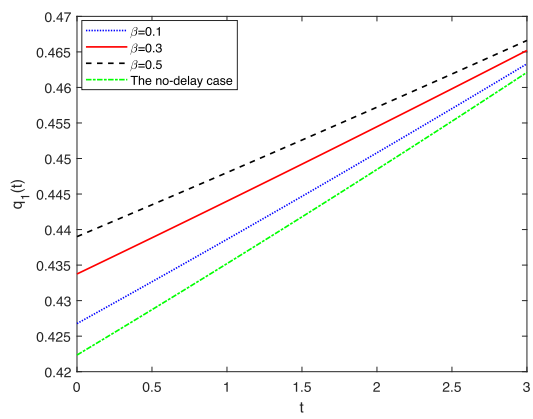

(h)

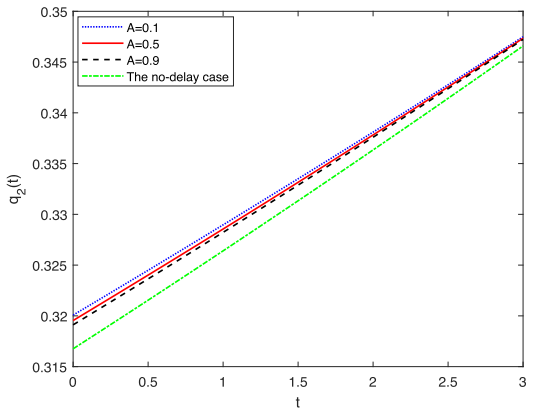

(c)

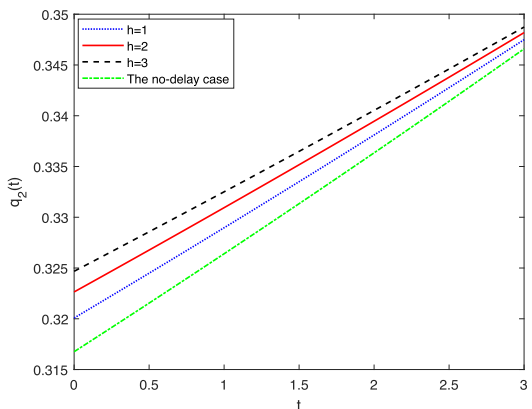

(f)

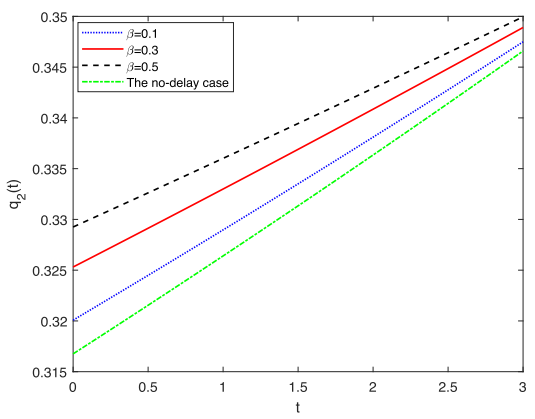

(i)

Figure 1 . The effect of $A, h$ and $\beta$ on robust equilibrium strategy.

wealth, the insurer increases the amount invested in risky assets when $p_{1}^{*}(t)$ is large. In Figures $1 \mathrm{~h}$ and $1 \mathrm{i}$, just as $\beta$ affects the robust equilibrium investment strategy, we can find that the larger $\beta$ is, the larger retention $q_{1}^{*}(t)$ and $q_{2}^{*}(t)$ are. From Figure 1, we can also find that the robust equilibrium investment and reinsurance strategy under the case of without delay is minimal. Note that in Corollary 3.7 it is mentioned that the model degenerates to the no-delay case only when $A=h=\beta=0$, not just when $A=0$. Thus, there is no contradiction between the robust equilibrium investment and reinsurance strategy minimized on the no-delay case and the robust equilibrium investment and reinsurance strategy decreasing with $A$.

Figure 2 demonstrates that the larger $\omega$ is, the smaller robust equilibrium investment and reinsurance strategy are. This is consistent with the financial implications of the risk aversion coefficient $\omega$. 


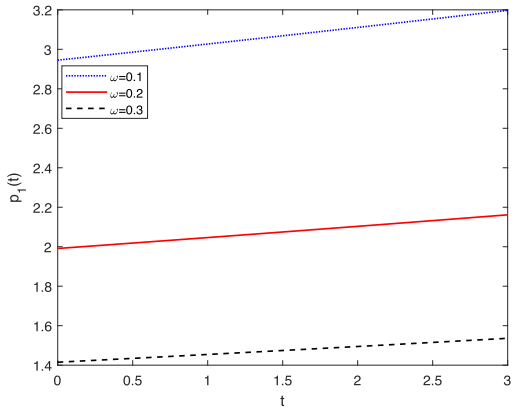

(a)

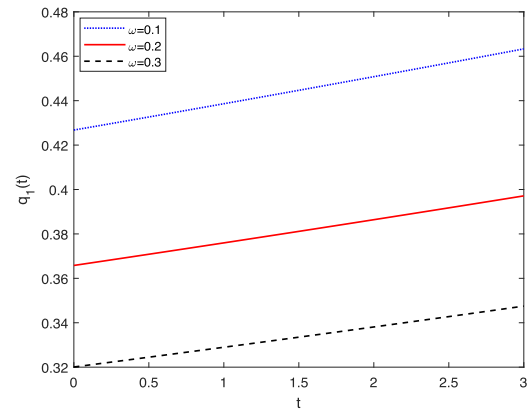

(b)

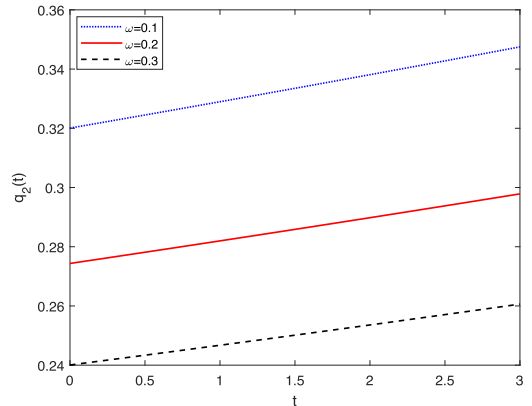

(c)

Figure 2. The effect of $\omega$ on robust equilibrium strategy.

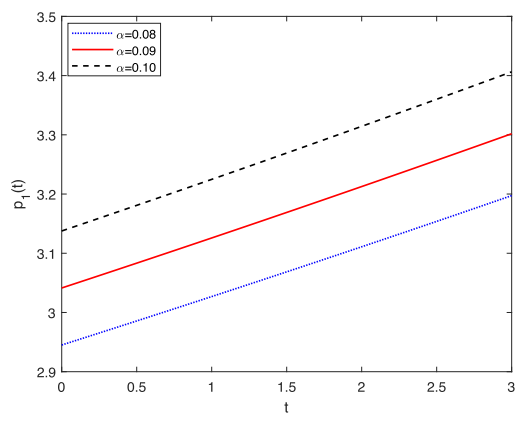

(a)

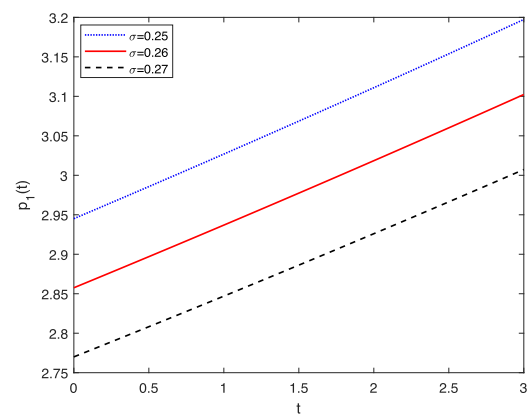

(b)

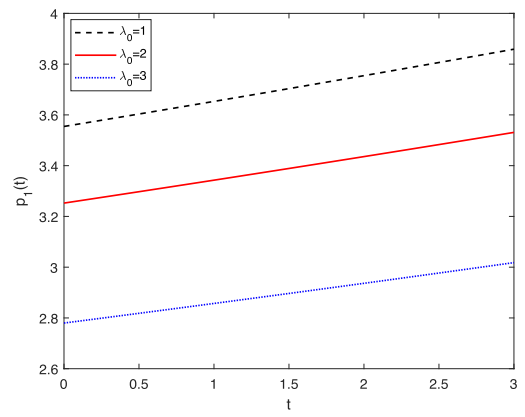

(c)

Figure 3 . The effect of $\alpha, \sigma$ and $\lambda_{0}$ on robust equilibrium investment strategy.

Figure 3 shows the effects of $\alpha, \sigma$ and $\lambda_{0}$ on robust equilibrium investment strategy $p_{1}^{*}(t)$. From Figure 3 a, we can find that $p_{1}^{*}(t)$ is increasing with the parameter $\alpha$. Since the value of $\alpha$ is increasing, the expected return rate of the risky asset is increasing, and thus the insurer would invest more money in the risky asset to gain more revenue. Figure 3b demonstrates that $p_{1}^{*}(t)$ decreases with $\sigma$. In fact, the bigger the value of $\sigma$ is, the larger the instantaneous volatility $\sigma$ of the risky asset is, which means the more risk of investment, and thus the insurer should invest less money in the risky asset. From Figure 3c, it is easy to see that the robust equilibrium investment strategy $p_{1}^{*}(t)$ is a decreasing function of $\lambda_{2}$, i.e., the insurer will invest less in the risky asset. Actually, the increase of the jump intensity $\lambda_{2}$ makes the risky asset more risk and less attractive.

Figure 4 depicts the effects of $\lambda, \lambda_{1}$ and $\lambda_{2}$ on robust equilibrium reinsurance strategy $q_{1}^{*}(t)$ and $q_{2}^{*}(t)$, respectively. As $\lambda$ increases, the average claim for the two businesses increases. To keep the claims risk at the expected level, the insurer increases its reinsurance purchases, i.e., retention $q_{1}^{*}(t)\left(q_{2}^{*}(t)\right)$ decreases, as shown in Figure 4a. From Figure $4 \mathrm{~b}$, we find that $q_{1}^{*}(t)$ increases with increase while $q_{2}^{*}(t)$ decreases. The average claim for the first line of business increases with $\lambda_{1}$, so the insurer increases its reinsurance purchases for the first line of business. In contrast, the second line of business has a comparative advantage since the average claim remains unchanged, and the insurer thus reduces the reinsurance purchases of the second line of business. Figure $4 \mathrm{c}$ shows that $q_{1}^{*}(t)$ decreases with $\lambda_{2}$ while $q_{2}^{*}(t)$ increases, which the economic explanation is similar to the above analysis.

Figure 5 demonstrates the effect $\xi_{1}, \xi_{2}$ and $\xi_{3}$ on the robust equilibrium investment and reinsurance strategy. From Figures $5 \mathrm{a}$ and $5 \mathrm{~b}$, we can find that the amount invested in the risky asset decreases with $\xi_{2}$ or $\xi_{3}$. Since 


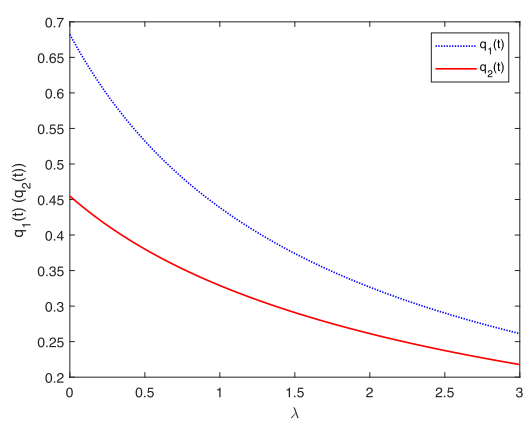

(a)

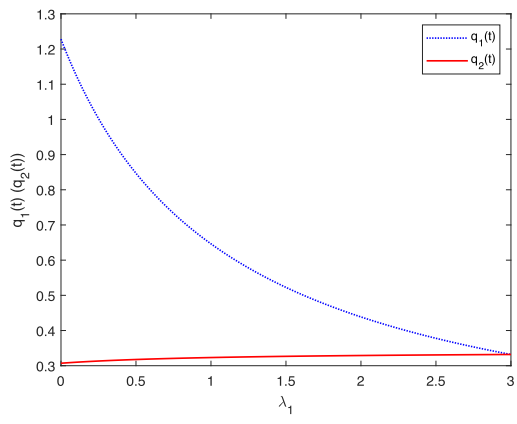

(b)

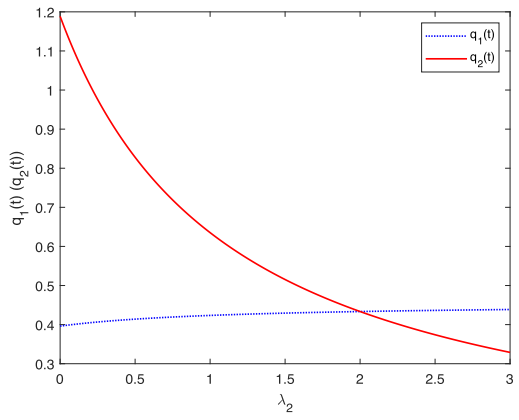

(c)

FiguRE 4 . The effect of $\lambda, \lambda_{1}$ and $\lambda_{2}$ on robust equilibrium reinsurance strategy.

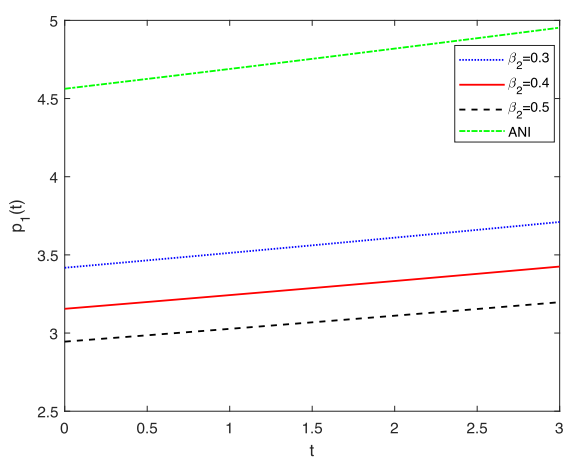

(a)

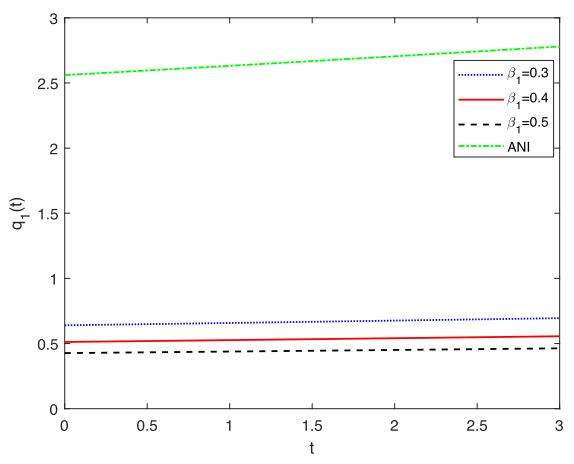

(c)

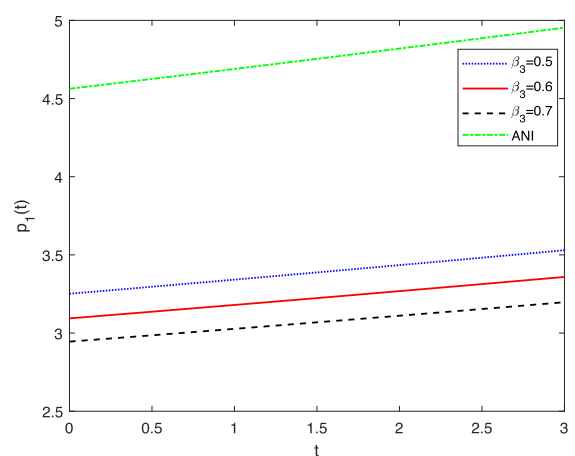

(b)

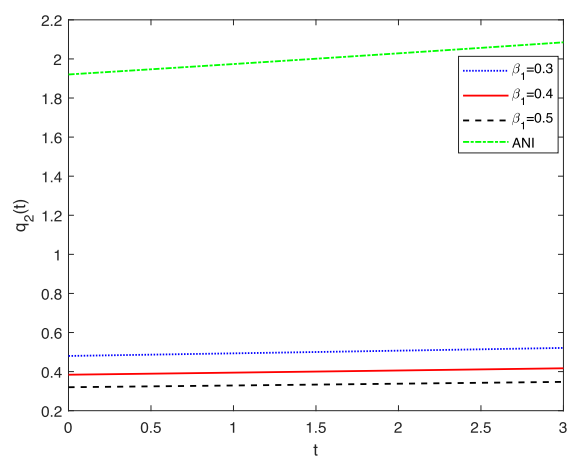

(d)

FiguRE 5 . The effect of $\xi_{1}, \xi_{2}$ and $\xi_{3}$ on robust equilibrium strategy

$\xi_{2}$ and $\xi_{3}$ denote the ambiguity aversion coefficient with respect to the diffusion risk and jump risk, respectively, the insurer has less confidence in the reference model with the larger $\xi_{2}$ or $\xi_{3}$, and thus the insurer will reduce the investment of the risky asset. Figures $5 \mathrm{c}$ and $5 \mathrm{~d}$ show that the larger the $\xi_{1}$, the smaller the retention of $q_{1}^{*}(t)$ and $q_{2}^{*}(t)$, with a similar mechanism of action to the effect of $\xi_{2}$ and $\xi_{3}$ on investment strategy. It is clear from Figure 5 that the investment and reinsurance strategy for ANI is significantly better than that for AAI. 


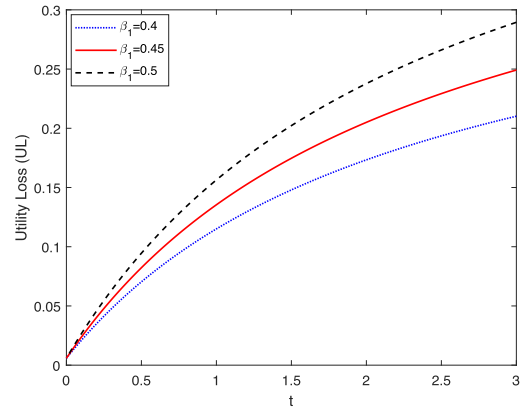

(a)

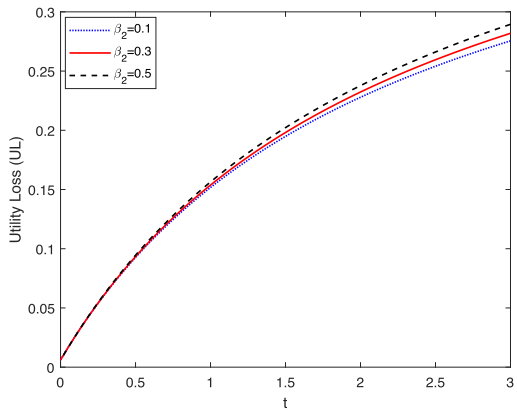

(b)

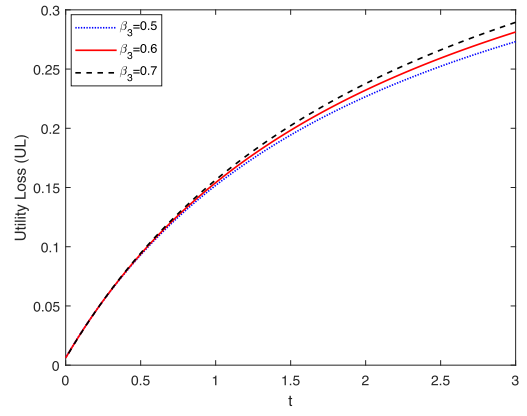

(c)

FiguRE 6 . The effect of $\xi_{1}, \xi_{2}$ and $\xi_{3}$ on utility loss.

Figure 6 demonstrates the utility loss from ignoring model uncertainty for the AAI. From Figure 6, we can find that the ambiguity aversion coefficients $\xi_{1}, \xi_{2}$ and $\xi_{3}$ have a positive impact on UL. That is, the utility loss is higher for the AAI with less information about reference measure $\mathbb{P}$ (larger $\xi_{1}, \xi_{2}$ and $\xi_{3}$ ) than for the AAI with more information about reference measure $\mathbb{P}$ (smaller $\xi_{1}, \xi_{2}$ and $\left.\xi_{3}\right)$.

\section{CONCLUSiOn}

In this paper, we consider a robust equilibrium investment and reinsurance problem with bounded memory and common shock dependence in a jump-diffusion financial market. The surplus process of the insurer is depicted by two-dimensional dependent claims, and the insurer is allowed to purchase proportional reinsurance. And we also assume that the insurer can invest his/her wealth in a risk-free asset and a risky asset satisfying jump-diffusion process. Next, we introduce the past performance and use it to derive the wealth process depicted by an SDDE. Applying the stochastic control theory under the framework of the game theory, together with stochastic control theory with delay, we derive an extended Hamilton-Jacobi-Bellman equation with delay. By solving the equation, we obtain a robust equilibrium strategy and the corresponding robust equilibrium value function. We also provide a numerical example to analyze the effects of delay parameters and risk dependent parameter on robust equilibrium strategy and explain why such effects occur. The main findings are as follows. (i) From the result of Theorem 3.2, we can find that the delay parameter has a significant effect on the robust equilibrium investment and reinsurance strategy, and its impact corresponds to the economic intuition. More specifically, according to the results of the numerical example, it is easy to see that the insurer can comprehensively consider the performance of a period of time since the introduction of the delay effect, and thus the insurer can enhance its risk control ability. And the insurer can adjust the parameters according to Remark 3.6 to improve risk control. (ii) The AAI's attitude towards ambiguity may impact his/her robust equilibrium investment and reinsurance strategy, such that the optimal investment and reinsurance strategy for the AAI facing model uncertainty is smaller than an ANI. (iii) The utility loss from ignoring model uncertainty increase with the ambiguity-aversion coefficients. (iv) The risk common shock factor has a significant impact on all reinsurance business.

In further research, some important factors affecting the insurer's decision may be introduced in the model, such as interest-rate risk, inflation risk, volatility risk, no-bankrupt constraint, and dynamic VaR constraint. However, the introduction of these factors will make the model more complicated. As a result, other methods, such as backward stochastic differential equations or other methods, may be introduced to solve the more complicated problem. 


\section{Appendix A. Derivation of Relative entropy}

The relative entropy is defined as the expectation under the alternative measure of the log Radon-Nikodym derivative defined in (2.7). From Itô's lemma, we derive

$$
\begin{aligned}
\mathrm{d} \ln \Lambda^{\varphi}(t)= & -\varphi_{1}(t) \mathrm{d} W_{0}(t)-\frac{1}{2} \varphi_{1}^{2}(t) \mathrm{d} t-\varphi_{2}(t) \mathrm{d} W(t)-\frac{1}{2} \varphi_{2}^{2}(t) \mathrm{d} t \\
& +\lambda_{0}\left(1-\varphi_{3}(t)\right) \mathrm{d} t+\int_{-1}^{\infty} \ln \varphi_{3}(t) N_{0}\left(\mathrm{~d} t, \mathrm{~d} y_{0}\right) .
\end{aligned}
$$

Then the relative entropy over the interval from $t$ to $t+\delta$ is as follows:

$$
\begin{aligned}
E^{\mathbb{Q}}\left[\ln \frac{\Lambda(t+\delta)}{\Lambda(t)}\right]= & E^{\mathbb{Q}}\left[-\int_{t}^{t+\delta} \varphi_{1}(u)\left(\mathrm{d} W_{0}^{\mathbb{Q}}(u)-\varphi_{1}(u) \mathrm{d} u\right)-\frac{1}{2} \int_{t}^{t+\delta} \varphi_{1}^{2}(u) \mathrm{d} u\right. \\
& -\int_{t}^{t+\delta} \varphi_{2}(u)\left(\mathrm{d} W^{\mathbb{Q}}(u)-\varphi_{2}(u) \mathrm{d} u\right)-\frac{1}{2} \int_{t}^{t+\delta} \varphi_{2}^{2}(u) \mathrm{d} u \\
& +\int_{t}^{t_{\delta}} \lambda_{0}\left(1-\varphi_{3}(u)\right) \mathrm{d} u+\int_{t}^{t+\delta} \int_{-1}^{\infty} \ln \varphi_{3}(u) \widetilde{N}_{0}\left(\mathrm{~d} u, \mathrm{~d} y_{0}\right) \\
& \left.+\int_{t}^{t+\delta} \int_{-1}^{\infty} \lambda_{0} \varphi_{3}(u) \ln \varphi_{3}(u) \mathrm{d} u\right] \\
= & E^{\mathbb{Q}}\left[\int_{t}^{t+\delta}\left(\frac{1}{2}\left(\varphi_{1}^{2}(u)+\varphi_{2}^{2}(u)\right)+\lambda_{0}\left(\varphi_{3}(u) \ln \varphi_{3}(u)-\varphi_{3}(u)+1\right) \mathrm{d} u\right)\right] .
\end{aligned}
$$

Let $\delta \rightarrow 0$, then $(2.10)$ is derived.

\section{Appendix B. Proof of Lemma 3.1}

Using Cauchy-Schwarz inequality, we have

$$
\left(\lambda_{1}+\lambda\right) E\left[Y_{1 i}^{2}\right]\left(\lambda_{2}+\lambda\right) E\left[Y_{2 i}^{2}\right] \geq\left(\sqrt{\left(\lambda+\lambda_{1}\right)\left(\lambda+\lambda_{2}\right)} E\left[Y_{1 i}\right] E\left[Y_{2 i}\right]\right)^{2}>\left(\lambda E\left[Y_{1 i}\right] E\left[Y_{2 i}\right]\right)^{2} .
$$

By $E\left[Y_{1 i}^{2}\right]>\left(E\left[Y_{1 i}\right]\right)^{2}, E\left[Y_{2 i}^{2}\right]>\left(E\left[Y_{2 i}\right]\right)^{2}$ and (2.1), we derive

$$
b_{1}^{2} b_{2}^{2}>\lambda^{2} \mu_{11}^{2} \mu_{21}^{2} .
$$

Moreover, we also have

$$
\begin{aligned}
\frac{\lambda \mu_{11} \mu_{21}}{b_{2}^{2}} \frac{a_{2}}{a_{1}} & =\frac{\lambda \mu_{11} \mu_{21}}{\left(\lambda+\lambda_{2}\right) \mu_{22}} \frac{\left(\lambda+\lambda_{2}\right) \mu_{21}}{\left(\lambda+\lambda_{1}\right) \mu_{11}}=\frac{\lambda \mu_{21}^{2}}{\left(\lambda+\lambda_{1}\right) \mu_{22}}, \\
\frac{b_{1}^{2}}{\lambda \mu_{11} \mu_{21}} \frac{a_{2}}{a_{1}} & =\frac{\left(\lambda+\lambda_{1}\right) \mu_{12}}{\lambda \mu_{11} \mu_{21}} \frac{\left(\lambda+\lambda_{2}\right) \mu_{21}}{\left(\lambda+\lambda_{1}\right) \mu_{11}}=\frac{\left(\lambda+\lambda_{2}\right) \mu_{12}}{\lambda \mu_{11}^{2}} .
\end{aligned}
$$

Noting that $\mu_{22}>\mu_{21}^{2}$ and $\mu_{12}>\mu_{11}^{2}$, then we have $\frac{\lambda \mu_{21}^{2}}{\left(\lambda+\lambda_{1}\right) \mu_{22}}<1$ and $\frac{\left(\lambda+\lambda_{2}\right) \mu_{12}}{\lambda \mu_{11}^{2}}>1$. Thus, equation (3.2) holds. 


\section{Appendix C. Proof of Theorem 3.2}

By the terminal condition of $V$ and $g$, we conjecture that $V$ and $g$ are of the following form:

$$
\begin{aligned}
V(t, x, l) & =H(t)(x+\beta l)+F(t), \\
g(t, x, l) & =P(t)(x+\beta l)+Q(t),
\end{aligned}
$$

where $H(T)=P(T)=1$ and $F(T)=Q(T)=0$.

Differentiating $V$ and $g$ with respect to $t, x$ and $l$, we obtain

$$
\begin{aligned}
& V_{t}=H^{\prime}(t)(x+\beta l)+F^{\prime}(t), \quad V_{x}=H(t), \quad V_{l}=\beta H(t)=\beta V_{x}, \quad V_{x x}=0, \\
& g_{t}=P^{\prime}(t)(x+\beta l)+Q^{\prime}(t), \quad g_{x}=P(t), \quad g_{l}=\beta P(t)=\beta g_{x}, \quad g_{x x}=0 .
\end{aligned}
$$

After simple calculations, we can also get

$$
\left\{\begin{array}{c}
E\left[V\left(t, x+p_{1} y_{0}, l\right)-V(t, x, l)\right]=\mu_{01} p_{1} H(t), \\
E\left[g^{2}\left(t, x+p_{1} y_{0}, l\right)-g^{2}(t, x, l)\right]=\mu_{02} p_{1}^{2} P^{2}(t) \\
\quad+2 \mu_{01} p_{1} P(t)[P(t)(x+\beta l)+Q(t)], \\
E\left[g\left(t, x+p_{1} y_{0}, l\right)-g(t, x, l)\right]=\mu_{01} p_{1} P(t) .
\end{array}\right.
$$

Noting that (2.6) and substituting the above results into (3.1), we derive

$$
\begin{aligned}
& \sup _{\pi \in \Pi} \inf _{\mathbb{Q} \in \mathcal{Q}}\left\{H^{\prime}(t)(x+\beta l)+F^{\prime}(t)+\left[\left(r-\gamma_{1}-\gamma_{2}+\beta\right)(x+\beta l)+(\alpha-r) p_{1}\right.\right. \\
& \quad+\left(\theta_{1}-\eta_{1}+q_{1} \eta_{1}\right) a_{1}+\left(\theta_{2}-\eta_{2}+q_{2} \eta_{2}\right) a_{2}-\sqrt{b_{1}^{2} q_{1}^{2}+b_{2}^{2} q_{2}^{2}+2 q_{1} q_{2} \lambda \mu_{11} \mu_{21}} \varphi_{1} \\
& \left.\quad-\sigma p_{1} \varphi_{2}+\lambda_{0} \varphi_{3} \mu_{01} p_{1}\right] H(t)-\frac{\omega}{2}\left(b_{1}^{2} q_{1}^{2}+b_{2}^{2} q_{2}^{2}+2 q_{1} q_{2} \lambda \mu_{11} \mu_{21}+p_{1}^{2} \sigma^{2}\right. \\
& \left.\left.\quad+\lambda_{0} \varphi_{3} \mu_{02} p_{1}^{2}\right) P^{2}(t)+\frac{\varphi_{1}^{2}}{2 \xi_{1}}+\frac{\varphi_{2}^{2}}{2 \xi_{2}}+\frac{\lambda_{0}\left(\varphi_{3} \ln \varphi_{3}-\varphi_{3}+1\right)}{\xi_{3}}\right\}=0, \\
& P^{\prime}(t)(x+\beta l)+Q^{\prime}(t)+\left[\left(r-\gamma_{1}-\gamma_{2}+\beta\right)(x+\beta l)+(\alpha-r) p_{1}^{*}+\left(\theta_{1}-\eta_{1}+q_{1}^{*} \eta_{1}\right) a_{1}\right. \\
& \quad+\left(\theta_{2}-\eta_{2}+q_{2}^{*} \eta_{2}\right) a_{2}-\sqrt{b_{1}^{2}\left(q_{1}^{*}\right)^{2}+b_{2}^{2}\left(q_{2}^{*}\right)^{2}+2 q_{1}^{*} q_{2}^{*} \lambda \mu_{11} \mu_{21} \varphi_{1}^{*}} \\
& \left.\quad-\sigma p_{1}^{*} \varphi_{2}^{*}+\lambda_{0} \varphi_{3}^{*} \mu_{01} p_{1}^{*}\right] P(t)=0 .
\end{aligned}
$$

Applying the first-order optimality conditions, $\varphi_{1}^{*}, \varphi_{2}^{*}$ and $\varphi_{3}^{*}$ which reach the infimum part in equation (C.3) are derived as follows:

$$
\begin{aligned}
& \varphi_{1}^{*}=\xi_{1} \sqrt{b_{1}^{2} q_{1}^{2}+b_{2}^{2} q_{2}^{2}+2 q_{1} q_{2} \lambda \mu_{11} \mu_{21}} H(t), \\
& \varphi_{2}^{*}=\xi_{2} \sigma p_{1} H(t), \\
& \varphi_{3}^{*}=e^{\xi_{3}\left(\frac{\omega}{2} \mu_{02} p_{1}^{2} P^{2}(t)-\mu_{01} p_{1} H(t)\right) .} .
\end{aligned}
$$


Substituting the above results back into (C.3), we derive

$$
\begin{aligned}
& \sup _{\pi \in \Pi}\left\{H^{\prime}(t)(x+\beta l)+F^{\prime}(t)+\left[\left(r-\gamma_{1}-\gamma_{2}+\beta\right)(x+\beta l)+(\alpha-r) p_{1}\right.\right. \\
& \left.\quad+\left(\theta_{1}-\eta_{1}+q_{1} \eta_{1}\right) a_{1}+\left(\theta_{2}-\eta_{2}+q_{2} \eta_{2}\right) a_{2}\right] H(t)-\frac{\omega}{2}\left(b_{1}^{2} q_{1}^{2}+b_{2}^{2} q_{2}^{2}+2 q_{1} q_{2} \lambda \mu_{11} \mu_{21}\right. \\
& \left.\quad+p_{1}^{2} \sigma^{2}\right) P^{2}(t)-\frac{\xi_{1}}{2}\left(b_{1}^{2} q_{1}^{2}+b_{2}^{2} q_{2}^{2}+2 q_{1} q_{2} \lambda \mu_{11} \mu_{21}\right) H^{2}(t)-\frac{\xi_{2}}{2} \sigma^{2} p_{1}^{2} H^{2}(t) \\
& \left.\quad+\frac{\lambda_{0}}{\xi_{3}}\left(1-e^{\xi_{3}\left(\frac{\omega}{2} \mu_{02} p_{1}^{2} P^{2}(t)-\mu_{01} p_{1} H(t)\right)}\right)\right\}=0 .
\end{aligned}
$$

Applying again the first-order optimality conditions, the optimal strategy $p_{1}^{*}, q_{1}^{*}$ and $q_{2}^{*}$ which reach the supremum part above equation are as follows:

$$
\begin{aligned}
p_{1}^{*}(t)= & \frac{1}{\omega P^{2}(t)+\xi_{2} H^{2}(t)}\left[\frac{\alpha-r}{\sigma^{2}} H(t)-\frac{\lambda_{0}}{\sigma^{2}}\left(\omega \mu_{02} p_{1}^{*}(t) P^{2}(t)\right.\right. \\
& \left.-\mu_{01} H(t)\right) e^{\left.\xi_{3}\left(\frac{\omega}{2} \mu_{02}\left(p_{1}^{*}(t)\right)^{2} P^{2}(t)-\mu_{01} p_{1}^{*}(t) H(t)\right)\right]}, \\
q_{1}^{*}(t)= & n_{1} \frac{H(t)}{\omega P^{2}(t)+\xi_{1} H^{2}(t)}, \\
q_{2}^{*}(t)= & n_{2} \frac{H(t)}{\omega P^{2}(t)+\xi_{1} H^{2}(t)}
\end{aligned}
$$

where

$$
n_{1}=\frac{a_{1} \eta_{1} b_{2}^{2}-a_{2} \eta_{2} \lambda \mu_{11} \mu_{21}}{b_{1}^{2} b_{2}^{2}-\lambda^{2} \mu_{11}^{2} \mu_{21}^{2}}, \quad n_{2}=\frac{a_{2} \eta_{2} b_{1}^{2}-a_{1} \eta_{1} \lambda \mu_{11} \mu_{21}}{b_{1}^{2} b_{2}^{2}-\lambda^{2} \mu_{11}^{2} \mu_{21}^{2}} .
$$

Substituting (C.5)-(C.7) and (C.9)-(C.11) back into (C.8) and (C.4), we derive

$$
\begin{aligned}
& H^{\prime}(t)(x+\beta l)+F^{\prime}(t)+\left[\left(r-\gamma_{1}-\gamma_{2}+\beta\right)(x+\beta l)+(\alpha-r) p_{1}^{*}+\left(\theta_{1}-\eta_{1}\right) a_{1}\right. \\
& \left.+\left(\theta_{2}-\eta_{2}\right) a_{2}\right] H(t)+\left(a_{1} n_{1} \eta_{1}+a_{2} n_{2} \eta_{2}-\frac{1}{2} b_{1}^{2} b_{1}^{2}-\frac{1}{2} b_{2}^{2} n_{2}^{2}-\lambda \mu_{11} \mu_{21} n_{1} n_{2}\right) \\
& \times\left(\frac{H^{2}(t)}{\omega P^{2}(t)+\xi_{1} H^{2}(t)}\right)-\frac{\left(p_{1}^{*}\right)^{2} \sigma^{2}}{2}\left(\omega P^{2}(t)+\xi_{2} H^{2}(t)\right) \\
& +\frac{\lambda_{0}}{\xi_{3}}\left(1-e^{\xi_{3}\left(\frac{\omega}{2} \mu_{02} p_{1}^{2} P^{2}(t)-\mu_{01} p_{1} H(t)\right)}\right)=0, \\
& P^{\prime}(t)(x+\beta l)+Q^{\prime}(t)+\left[\left(r-\gamma_{1}-\gamma_{2}+\beta\right)(x+\beta l)+(\alpha-r) p_{1}^{*}+\left(\theta_{1}-\eta_{1}\right) a_{1}\right. \\
& \left.+\left(\theta_{2}-\eta_{2}\right) a_{2}\right] P(t)+\left(a_{1} n_{1} \eta_{1}+a_{2} n_{2} \eta_{2}\right) \frac{H(t) P(t)}{\omega P^{2}(t)+\xi_{1} H^{2}(t)} \\
& -\xi_{1}\left(b_{1}^{2} n_{1}^{2}+b_{2}^{2} n_{2}^{2}+2 n_{1} n_{2} \lambda \mu_{11} \mu_{21}\right) \frac{H^{3}(t) P(t)}{\left(\omega P^{2}(t)+\xi_{1} H^{2}(t)\right)^{2}} \\
& -\xi_{2} \sigma^{2}\left(p_{1}^{*}\right)^{2} H(t) P(t)+\lambda_{0} \mu_{01} p_{1}^{*} P(t) e^{\xi_{3}\left(\frac{\omega}{2} \mu_{02}\left(p_{1}^{*}\right)^{2} P^{2}(t)-\mu_{01} p_{1}^{*} H(t)\right)}=0 .
\end{aligned}
$$

Matching the coefficient on the both sides, we can obtain the following differential equations:

$$
\begin{aligned}
H^{\prime}(t)+\left(r-\gamma_{1}-\gamma_{2}+\beta\right) H(t)=0, & H(T)=1, \\
P^{\prime}(t)+\left(r-\gamma_{1}-\gamma_{2}+\beta\right) P(t)=0, & P(T)=1,
\end{aligned}
$$




$$
\begin{aligned}
F^{\prime}(t) & +\left[(\alpha-r) p_{1}^{*}+\left(\theta_{1}-\eta_{1}\right) a_{1}+\left(\theta_{2}-\eta_{2}\right) a_{2}\right] H(t)-\frac{\left(p_{1}^{*}\right)^{2} \sigma^{2}}{2}\left(\omega P^{2}(t)+\xi_{2} H^{2}(t)\right) \\
& +\frac{\lambda_{0}}{\xi_{3}}\left(1-e^{\xi_{3}\left(\frac{\omega}{2} \mu_{02}\left(p_{1}^{*}\right)^{2} P^{2}(t)-\mu_{01} p_{1}^{*} H(t)\right)}\right)+\left(a_{1} n_{1} \eta_{1}+a_{2} n_{2} \eta_{2}-\frac{1}{2} b_{1}^{2} n_{1}^{2}-\frac{1}{2} b_{2}^{2} n_{2}^{2}\right. \\
& \left.-\lambda \mu_{11} \mu_{21} n_{1} n_{2}\right) \frac{1}{\omega+\xi_{1}}=0, \quad F(T)=0, \\
Q^{\prime}(t) & +\left[(\alpha-r) p_{1}^{*}+\left(\theta_{1}-\eta_{1}\right) a_{1}+\left(\theta_{2}-\eta_{2}\right) a_{2}\right] P(t)-\xi_{2} \sigma^{2}\left(p_{1}^{*}\right)^{2} H(t) P(t) \\
& +\lambda_{0} \mu_{01} p_{1}^{*} P(t) e^{\xi_{3}\left(\frac{\omega}{2} \mu_{02}\left(p_{1}^{*}\right)^{2} P^{2}(t)-\mu_{01} p_{1}^{*} H(t)\right)}+\left(a_{1} n_{1} \eta_{1}+a_{2} n_{2} \eta_{2}\right) \frac{1}{\omega+\xi_{1}} \\
& -\xi_{1}\left(b_{1}^{2} n_{1}^{2}+b_{2}^{2} n_{2}^{2}+2 \lambda \mu_{11} \mu_{21} n_{1} n_{2}\right) \frac{1}{\left(\omega+\xi_{1}\right)^{2}}=0, \quad Q(T)=0 .
\end{aligned}
$$

Considering the terminal value condition $H(T)=P(T)=1$ and $F(T)=Q(T)=0$, the above equations can be solved as

$$
\begin{aligned}
& H(t)=P(t)=e^{\left(r-\gamma_{1}-\gamma_{2}+\beta\right)(T-t)}, \\
& F(t)=-\frac{\left[\left(\theta_{1}-\eta_{1}\right) a_{1}+\left(\theta_{2}-\eta_{2}\right) a_{2}\right]}{r-\gamma_{1}-\gamma_{2}+\beta}\left(1-e^{\left(r-\gamma_{1}-\gamma_{2}+\beta\right)(T-t)}\right) \\
& +\frac{1}{\omega+\xi_{1}}\left(a_{1} n_{1} \eta_{1}+a_{2} n_{2} \eta_{2}-\frac{1}{2} b_{1}^{2} n_{1}^{2}-\frac{1}{2} b_{2}^{2} n_{2}^{2}-\lambda \mu_{11} \mu_{21} n_{1} n_{2}\right)(T-t) \\
& +\int_{t}^{T}\left[(\alpha-r) p_{1}^{*}(s) e^{\left(r-\gamma_{1}-\gamma_{2}+\beta\right)(T-s)}\right. \\
& -\frac{\left(p_{1}^{*}(s)\right)^{2} \sigma^{2}\left(\omega+\xi_{2}\right) e^{2\left(r-\gamma_{1}-\gamma_{2}+\beta\right)(T-s)}}{2} \\
& \left.+\frac{\lambda_{0}}{\xi_{3}}\left(1-e^{\xi_{3}\left(\frac{\omega}{2} \mu_{02}\left(p_{1}^{*}(s)\right)^{2} e^{2\left(r-\gamma_{1}-\gamma_{2}+\beta\right)(T-s)}-\mu_{01}\left(p_{1}^{*}(s)\right)^{2} e^{\left(r-\gamma_{1}-\gamma_{2}+\beta\right)(T-s)}\right)}\right)\right] \mathrm{d} s, \\
& Q(t)=-\frac{\left[\left(\theta_{1}-\eta_{1}\right) a_{1}+\left(\theta_{2}-\eta_{2}\right) a_{2}\right]}{r-\gamma_{1}-\gamma_{2}+\beta}\left(1-e^{\left(r-\gamma_{1}-\gamma_{2}+\beta\right)(T-t)}\right) \\
& +\frac{1}{\omega+\xi_{1}}\left(a_{1} n_{1} \eta_{1}+a_{2} n_{2} \eta_{2}\right)(T-t) \\
& -\frac{\xi_{1}}{\left(\omega+\xi_{1}\right)^{2}}\left(b_{1}^{2} n_{1}^{2}+b_{2}^{2} n_{2}^{2}+2 \lambda \mu_{11} \mu_{21} n_{1} n_{2}\right)(T-t) \\
& +\int_{t}^{T}\left[(\alpha-r) p_{1}^{*}(s)-\xi_{2} \sigma^{2}\left(p_{1}^{*}(s)\right)^{2} e^{2\left(r-\gamma_{1}-\gamma_{2}+\beta\right)(T-s)}\right. \\
& +\lambda_{0} \mu_{01} p_{1}^{*}(s) e^{\left(r-\gamma_{1}-\gamma_{2}+\beta\right)(T-s)}
\end{aligned}
$$

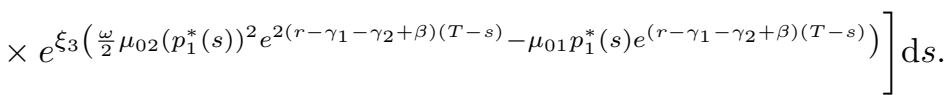

Substituting the above results back into (C.9)-(C.11), (C.1) and (C.5)-(C.7), we obtain (3.3)-(3.9). Noting that Case 2 (i.e., $n_{1}>0$ and $n_{2}>0$ ), it is easy to see that $q_{1}^{*}(t)>0$ and $q_{2}^{*}(t)>0$. Then, the proof of Theorem 3.2 is completed.

\section{Appendix D. Proof of Proposition 3.3}

From equation (3.3), we can derive 


$$
\begin{aligned}
\sigma^{2} p_{1}^{*}\left(\omega+\xi_{2}\right) e^{\left(r-\gamma_{1}-\gamma_{2}+\beta\right)(T-t)}= & \alpha-r-\lambda_{0}\left(\omega \mu_{02} p_{1}^{*} e^{\left(r-\gamma_{1}-\gamma_{2}+\beta\right)(T-t)}-\mu_{01}\right) \\
& \times e^{\xi_{3}\left(\frac{\omega}{2} \mu_{02}\left(p_{1}^{*}\right)^{2} e^{2\left(r-\gamma_{1}-\gamma_{2}+\beta\right)(T-t)}-\mu_{01} p_{1}^{*} e^{\left(r-\gamma_{1}-\gamma_{2}+\beta\right)(T-t)}\right) .} .
\end{aligned}
$$

Let

$$
\begin{aligned}
h\left(p_{1}\right)= & \alpha-r-\lambda_{0}\left(\omega \mu_{02} p_{1} e^{\left(r-\gamma_{1}-\gamma_{2}+\beta\right)(T-t)}-\mu_{01}\right) \\
& \times e^{\xi_{3}\left(\frac{\omega}{2} \mu_{02} p_{1}^{2} e^{2\left(r-\gamma_{1}-\gamma_{2}+\beta\right)(T-t)}-\mu_{01} p_{1} e^{\left(r-\gamma_{1}-\gamma_{2}+\beta\right)(T-t)}\right)}-\sigma^{2} p_{1}\left(\omega+\xi_{2}\right) e^{\left(r-\gamma_{1}-\gamma_{2}+\beta\right)(T-t)} .
\end{aligned}
$$

Further, the derivative of $h(\cdot)$ is as follows:

$$
\begin{aligned}
h^{\prime}\left(p_{1}\right)= & -\lambda_{0} \omega \mu_{02} e^{\left(r-\gamma_{1}-\gamma_{2}+\beta\right)(T-t)} e^{\xi_{3}\left(\frac{\omega}{2} \mu_{02} p_{1}^{2} e^{2\left(r-\gamma_{1}-\gamma_{2}+\beta\right)(T-t)}-\mu_{01} p_{1} e^{\left(r-\gamma_{1}-\gamma_{2}+\beta\right)(T-t)}\right)} \\
& -\lambda_{0} \xi_{3} e^{\left(r-\gamma_{1}-\gamma_{2}+\beta\right)(T-t)}\left(\omega \mu_{02} p_{1} e^{\left(r-\gamma_{1}-\gamma_{2}+\beta\right)(T-t)}-\mu_{01}\right)^{2} \\
& \times e^{\xi_{3}\left(\frac{\omega}{2} \mu_{02} p_{1}^{2} e^{2\left(r-\gamma_{1}-\gamma_{2}+\beta\right)(T-t)}-\mu_{01} p_{1} e^{\left(r-\gamma_{1}-\gamma_{2}+\beta\right)(T-t)}\right)} \\
& -\sigma^{2}\left(\omega+\xi_{2}\right) e^{\left(r-\gamma_{1}-\gamma_{2}+\beta\right)(T-t)}<0
\end{aligned}
$$

that is, $h\left(p_{1}\right)$ is a decreasing function with respect to $p_{1}$. In addition, it is easy to see that $h(0)=\alpha-r+\lambda_{0} \mu_{01}>0$, and also $h\left(p_{1}\right)<0$ for $p_{1}>\max \left\{\frac{\mu_{01}}{\omega \mu_{02} e^{\left(r-\gamma_{1}-\gamma_{2}+\beta\right)(T-t)}}, \frac{\alpha-r}{\sigma^{2}\left(\omega+\xi_{2}\right) e^{\left(r-\gamma_{1}-\gamma_{2}+\beta\right)(T-t)}}\right\}>0$. The proof is completed.

Acknowledgements. This work is supported by the project of the Chengdu University of Information Technology Introducing Talents to Launch Scientific Research.

\section{REFERENCES}

[1] E.W. Anderson, L.P. Hansen and T.J. Sargent, A quartet of semigroups for model specification, robustness, prices of risk, and model detection. J. Eur. Econ. Assoc. 1 (2003) 68-123.

[2] L. Bai and J. Guo, Optimal proportional reinsurance and investment with multiple risky assets and no-shorting constraint. Insurance Math. Econ. 42 (2008) 968-975.

[3] L. Bai and H. Zhang, Dynamic mean-variance problem with constrained risk control for the insurers. Math. Methods Oper. Res. 68 (2008) 181-205.

[4] N. Bäuerle, Benchmark and mean-variance problems for insurers. Math. Methods Oper. Res. 62 (2005) $159-165$.

[5] J. Bi and J. Guo, Optimal mean-variance problem with constrained controls in a jump-diffusion financial market for an insurer. J. Optim. Theory App. 157 (2013) 252-275.

[6] T. Björk and A. Murgoci, A general theory of markovian time inconsistent stochastic control problems. Working paper, Stockholm School of School (2010).

[7] T. Björk, M. Khapko and A. Murgoci, On time-inconsistent stochastic control in continuous time. Finance Stochastics 21 (2017) 331-360.

[8] N. Branger and L.S. Larsen, Robust portfolio choice with uncertainty about jump and diffusion risk. J. Banking Finance 37 (2013) 5036-5047.

[9] S. Browne, Optimal investment policies for a firm with a random risk process: exponential utility and minimizing the probability of ruin. Math. Oper. Res. 20 (1995) 937-958.

[10] Y. Cao and N. Wan, Optimal proportional reinsurance and investment based on Hamilton-Jacobi-Bellman equation. Insurance Math. Econ. 45 (2009) 157-162.

[11] M.-H. Chang, T. Pang and Y. Yang, A stochastic portfolio optimization model with bounded memory. Math. Oper. Res. 36 (2011) 604-619.

[12] Z. Chen and P. Yang, Robust optimal reinsurance-investment strategy with price jumps and correlated claims. Insurance Math. Econ. 92 (2020) 27-46.

[13] J. Grandell, Aspects of Risk Theory. Springer-Verlag, New York (1991).

[14] C. Hipp and M. Plum, Optimal investment for insurers. Insurance Math. Econ. 27 (2000) 215-228.

[15] B. Hojgaard and M. Taksar, Optimal proportional reinsurance policies for diffusion models with transaction costs. Special issue on the interplay between insurance, finance and control. Insurance Math. Econ. 22 (1998) 41-51.

[16] D. Li and W.-L. Ng, Optimal dynamic portfolio selection: multiperiod mean-variance formulation. Math. Finance 10 (2000) $387-406$. 
[17] X. Lin and Y. Qian, Time-consistent mean-variance reinsurance-investment strategy for insurers under CEV model. Scand. Actuarial J. 2016 (2016) 646-671.

[18] P.J. Maenhout, Robust portfolio rules and detection-error probabilities for a mean-reverting risk premium. J. Econ. Theory 128 (2006) 136-163.

[19] H. Markowitz, Portfolio selection. J. Finance 7 (1952) 77-91.

[20] B. Øksendal and A. Sulem, A maximum principle for optimal control of stochastic systems with delay, with applications to finance. In: Optimal Control and Partial Differential Equations-Innovations and Applications, edited by J.M. Menaldi, E. Rofman and A. Sulem. IOS Press, Amsterdam, The Netherlands (2000) 64-79.

[21] H. Schmidli, Stochastic Control in Insurance. Springer-Verlag London (2008).

[22] Y. Shen and Y. Zeng, Optimal investment-reinsurance with delay for mean-variance insurers: a maximum principle approach. Insurance Math. Econ. 57 (2014) 1-12.

[23] Y. Shen, Q. Meng and P. Shi, Maximum principle for mean-field jump-diffusion stochastic delay differential equations and its application to finance. Automatica 50 (2014) 1565-1579.

[24] H. Yang and L. Zhang, Optimal investment for insurer with jump-diffusion risk process. Insurance Math. Econ. 37 (2005) 615-634.

[25] B. Yi, F. Viens, Z. Li and Y. Zeng, Robust optimal strategies for an insurer with reinsurance and investment under benchmark and mean-variance criteria. Scand. Actuarial J. 2015 (2015) 725-751.

[26] Y. Zeng and Z. Li, Optimal time-consistent investment and reinsurance policies for mean-variance insurers. Insurance Math. Econ. 49 (2011) 145-154.

[27] Y. Zeng, Z. Li and Y. Lai, Time-consistent investment and reinsurance strategies for mean-variance insurers with jumps. Insurance Math. Econ. 52 (2013) 498-507.

[28] Y. Zeng, D. Li and A. Gu, Robust equilibrium reinsurance-investment strategy for a mean-variance insurer in a model with jumps. Insurance Math. Econ. 66 (2016) 138-152.

[29] X.Y. Zhou and D. Li, Continuous-time mean-variance portfolio selection: a stochastic LQ framework. Appl. Math. Optim. 42 (2000) 19-33.

\section{Subscribe to Open (S2O) A fair and sustainable open access model}

This journal is currently published in open access under a Subscribe-to-Open model (S2O). S2O is a transformative model that aims to move subscription journals to open access. Open access is the free, immediate, online availability of research articles combined with the rights to use these articles fully in the digital environment. We are thankful to our subscribers and sponsors for making it possible to publish this journal in open access, free of charge for authors.

\section{Please help to maintain this journal in open access!}

Check that your library subscribes to the journal, or make a personal donation to the S2O programme, by contacting subscribers@edpsciences.org

More information, including a list of sponsors and a financial transparency report, available at: https://www. edpsciences.org/en/maths-s2o-programme 